\title{
The Role of Integer Matrices in Multidimensional Multirate Systems
}

\author{
Tsuhan Chen, Student Member, IEEE, and P. P. Vaidyanathan, Fellow, IEEE
}

\begin{abstract}
The basic building blocks in a multidimensional (MD) multirate system are the decimation matrix $M$ and the expansion matrix $L$. For the $D$-dimensional case these are $D \times$ $D$ nonsingular integer matrices. When these matrices are diagonal, most of the one-dimensional (1D) results can be extended automatically. However, for the nondiagonal case, these extensions are nontrivial. Some of these extensions, e.g. polyphase decomposition and maximally decimated perfect reconstruction systems, have already been successfully made by some authors. However, there exist several 1D results in multirate processing, for which the multidimensional extensions are even more difficult. An example is the development of polyphase representation for rational (rather than integer) sampling rate alterations. In the $1 \mathrm{D}$ case, this development relies on the commutativity of decimators and expanders, which is possible whenever $M$ and $L$ are relatively prime (coprime). The conditions for commutativity in the two-dimensional (2D) case have recently been developed successfully in [1]. In the MD case, the results are more involved. In this paper we formulate and solve a number of problems of this nature. Our discussions are based on several key properties of integer matrices, including greatest common divisors and least common multiples, which we first review. These properties are analogous to those of polynomial matrices, some of which have been used in system theoretic work (e.g., matrix fraction descriptions, coprime matrices, Smith form, and so on).
\end{abstract}

\section{INTRODUCTION}

$\mathrm{R}$ ECENTLY, there has been much research work on multidimensional (MD) multirate systems, which have found applications in the processing of images and video data. The key building blocks in these systems are the decimation matrix $\boldsymbol{M}$ and the expansion matrix $\boldsymbol{L}$. With $D$ denoting the number of dimensions, these are $D \times D$ nonsingular integer matrices. An excellent review of MD multirate systems, along with many new developments in filter banks, is given in [2].

When these matrices are diagonal, most of the one-dimensional (1D) results can be extended automatically (by performing operation in each dimension separately). However, for the nondiagonal case, these extensions are nontrivial and require more complicated notations and matrix operations. Some of these extensions, e.g., polyphase decomposition and maximally decimated perfect

Manuscript received August 21, 1991; revised March 13, 1992. This work was supported in part by National Science Foundation Grants MIP 8604456 , MIP 8919196, and by matching funds from Tektronix, Inc., and Rockwell International.

The authors are with the Department of Electrical Engineering, California Institute of Technology, Pasadena, CA 91125

IEEE Log Number 9206033. reconstruction systems, have already been successfully made by some authors [2]-[11]. However, there still exist several 1D results in multirate processing, for which the multidimensional extensions are even more difficult. In this paper, we shall address some problems of this nature, including i) the development of polyphase representation for rational sampling rate alterations, ii) the perfect-reconstruction properties of MD delay-chain systems, and iii) the periodicity properties of decimated periodic signals. Some preliminary results have been reported by the authors in [12], [13].

The first of the above three problems can be solved provided a number of conditions are satisfied. This includes the commutativity of decimators and expanders. For the two-dimensional (2D) case, the commutativity issue has been successfully addressed recently in [1], where the commutativity is used in a different context, i.e., the design of 2D filter banks with arbitrary rational decimation matrices.

Before formulating and solving these problems, in Section II we first review fundamental concepts of MD multirate signal processing. In Section III, we introduce some properties about integer matrices.' These are crucial to our discussions because of the role played by the decimation and expansion matrices. Some of these properties are analogous to those of polynomial matrices, which have been well developed by researchers in system theory, e.g., matrix fraction descriptions, greatest common right/left divisors (gcrd/gcld), coprime matrices, Smith form, and so on [18], [17]. We also review the concepts of the least common right multiple (lcrm) and least common left multiple (lclm) of integer matrices [14, p. 35] and develop further their properties. We shall also present an approach to finding an $\mathrm{lcrm} / \mathrm{lclm}$ of two integer matrices, which is very useful in practical applications.

All of these will be applied in deriving many of the new multirate results summarized next.

\section{A. Polyphase Structures for Rational Sampling Rate Alterations (Section IV)}

In 1D, multirate techniques permit us to alter the sampling rate of a sequence by a rational fraction, e.g., to

'In fact, these properties can be applied to matrices with elements from a so-called "principle ideal domain" (pid) [14]-[17]. The set of integers and the set of polynomials with coefficients belonging to a field are two examples of pid's 
reduce the sampling rate by $M / L$. Fig. 1 shows a scheme to achieve this. Note that the filter $H(z)$ is used to suppress image components generated by the $L$-fold expander and to eliminate aliasing owing to the $M$-fold decimator as well. In MD, also it is often necessary to interface images (or video data) between systems which use different sampling methods (different sampling lattices) [19], [3]. The conversion between the European and North American television systems, and the conversion between high definition television (HDTV) signals and conventional television signals [20] are two examples.

For the 1D case, using the polyphase approach, we can implement Fig. 1 more efficiently as in either Fig. 2(a) or Fig. 2(b) (for the case $M=3$ and $L=2$ ). Then, it seems that we cannot improve the efficiency anymore because we cannot use Noble identities [21], [22] to move the expanders further to the right (or the decimators further to the left). However, it turns out that we can still do so by using the technique introduced in [23]. We shall refer to this technique as the rational polyphase implementation (RPI). Fig. 3 shows the development of the RPI technique by successively redrawing the rational decimation circuit. Starting from Fig. 2(a), we replace $z^{-1}$ by $z^{2} z^{-3}$ and $z^{-2}$ by $z^{4} z^{-6}$, so we get Fig. 3(a). Note that when $M$ and $L$ are relatively prime (coprime), we are able to express every integer $k$ as $L k_{1}+M k_{2}$ for some integers $k_{1}$ and $k_{2}$ (Euclid's theorem). With the help of Noble identities, Fig. 3(a) can be redrawn as Fig. 3(b). Next, we can interchange the expanders and decimators when $M$ and $L$ are coprime [21], and obtain Fig. 3(c). Finally we can perform Type 2 polyphase decomposition on $E_{i}(z)$ 's with respect to $L$, and get Fig. 3(d). In summary, Fig. 3(d) is equivalent to Fig. 2(a) but now each arithmetic operation is performed at its lowest rate. Note that the RPI technique works if and only if $M$ and $L$ are coprime.

New Results of Section IV: In Section IV, we will extend this polyphase technique for MD systems and show that the necessary and sufficient conditions for its feasibility are: 1) $\boldsymbol{M L}=\boldsymbol{L} \boldsymbol{M}$ (i.e., $\boldsymbol{M}$ and $\boldsymbol{L}$ commute) and 2) $M$ and $L$ are coprime. The coprimeness of matrices will be defined later in Section III. Note that, in general, we have to distinguish left coprimeness and right coprimeness for the matrix case. However, we will show that if $\boldsymbol{M} \boldsymbol{L}=\boldsymbol{L} \boldsymbol{M}$, left coprimeness and right coprimeness are equivalent.

We should point out that the conditions for commutativity of an $\boldsymbol{M}$-fold decimator and an $\boldsymbol{L}$-fold expander has been generalized to the $2 \mathrm{D}$ case in [1], where the conditions are given for upper triangular $\boldsymbol{M}$ and $\boldsymbol{L}$. In Section IV, we will present conditions which hold for any number of dimensions without any assumption on $\boldsymbol{M}$ and $\boldsymbol{L}$.

\section{B. Generalized Delay-Chain Systems (Section V)}

A 1D delay-chain system is shown in Fig. 4. It has been shown that this is a perfect-reconstruction (PR) system

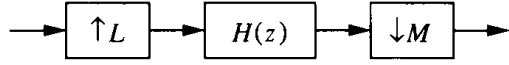

Fig. 1. One-dimensional rational decimation system

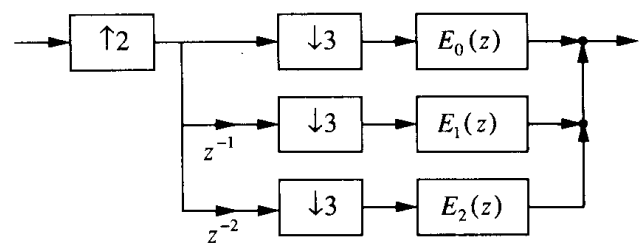

(a)

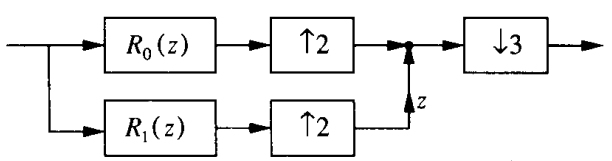

(b)

Fig. 2. Two types of polyphase implementations of a ID rational decimation system.
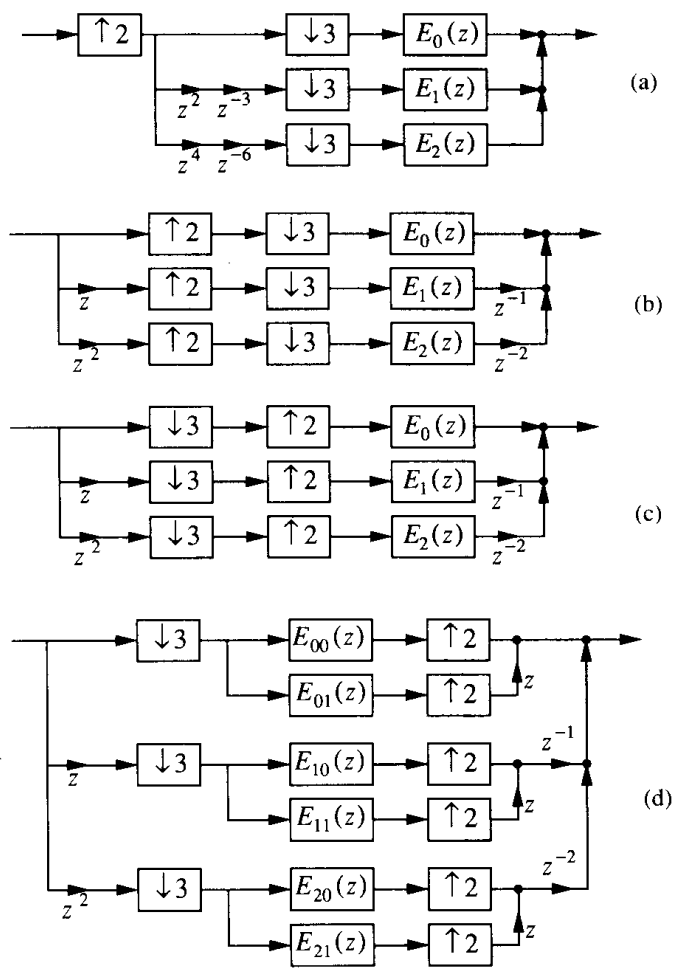

Fig. 3. Successive redrawing of polyphase implementations of a $1 \mathrm{D} \mathrm{ra-}$ tional decimation system.

(i.e., $\hat{x}(n)=x(n)$ ) if and only if $L$ and $M$ are coprime [24, lemma A.2]. This system is fundamental to many $1 \mathrm{D}$ maximally decimated PR filter banks [21]. The case where $L=1$ in Fig. 4 is most common. The case where $L \neq 1$ 


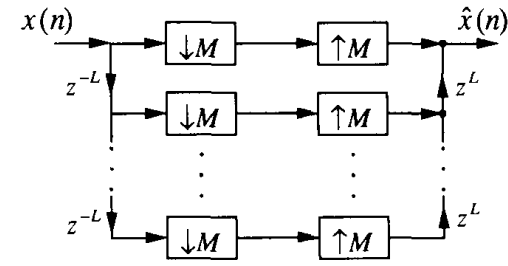

Fig. 4. One-dimensional delay-chain system.

is also required in some applications where pairs of analysis filters are constrained by symmetry conditions [24]. In Section V, we will consider the MD extension of Fig. 4 and then discuss the conditions for PR.

\section{Periodicity Matrices of Decimated Signals (Section VI)}

In the $1 D$ case, if a periodic signal $x(n)$ with period $L$ is decimated by a factor of $M$ to obtain $y(n)=x(M n)$, then the period of $y(n)$ is easily verified to be $L / \operatorname{gcd}(M$, $L)=\operatorname{lcm}(M, L) / M$. (If further information about $x(n)$ is available, then smaller periods can be found.) We shall extend this result to the MD case, where an MD signal $x(n)$ with periodicity matrix $L$ is decimated by the matrix $M$. We will show that the periodicity matrix of $y(n)=$ $x(\boldsymbol{M n})$ is $\boldsymbol{M}^{-1} \operatorname{lcrm}(\boldsymbol{M}, \boldsymbol{L})$, where $\operatorname{lcrm}(\boldsymbol{M}, \boldsymbol{L})$ denotes an lcrm of $\boldsymbol{M}$ and $\boldsymbol{L}$. We will also extend these results for stochastic signals. More specifically, assuming that $x(n)$ is cyclo-widesense stationary (CWSS) with periodicity matrix $L$, we will derive the periodicity matrix of cyclostationarity of $x(M n)$.

\section{Emerging Results from Other Authors (Section VIII)}

After we submitted this paper, we came to realize via private communication with a number of authors that several other groups were simultaneously arriving at similar and related results, particularly about the commutativity of decimators and expanders. In Section VIII, we provide mathematical details on these other contributions.

\section{Review of Basic Concepts}

We first review some basic concepts of MD multirate techniques, which are crucial to our discussion. More detailed discussion and derivations can be found in [25], [26], [3], [8], [2]. In particular, [2] contains an excellent review, including many of the notations we have summarized as follows. Some of the notations we use here are slightly modified versions of those in [2], and suit our discussions better.

1) Notations: Capital and lowercase boldfaced letters denote matrices and vectors, respectively. The symbol $I$ is reserved for the identity matrix. The notations $\boldsymbol{A}^{T}, \boldsymbol{A}^{-1}$, and $\boldsymbol{A}^{-T}$ denote the transpose, the inverse, and the inverse transpose of $\boldsymbol{A}$, respectively. The row and column indices typically begin from zero. Also,

$D$ : number of dimensions.

$\Re$ : set of all $D \times 1$ integer vectors.

$[a, b)^{D}$ : set of $D \times 1$ real vectors $\boldsymbol{x}$ with components $x_{i}$ in the range $a \leq x_{i}<b$.

$\boldsymbol{n}=\left[n_{0} n_{1} \cdots n_{D-1}\right]^{T}$ : "time" index of MD discrete signals. All $n_{i}$ 's are integer, so that $\boldsymbol{n} \in \mathfrak{R}$.

$\boldsymbol{\omega}=\left[\omega_{0} \omega_{1} \cdots \omega_{D-1}\right]^{T}:$ frequency variable of the Fourier transform of MD discrete signals.

$x(\boldsymbol{n}) \leftrightarrow X_{F}(\boldsymbol{\omega})$ : Fourier transform pair, defined as

$$
\begin{aligned}
X_{F}(\boldsymbol{\omega}) & =\sum_{n \in \Re} x(\boldsymbol{n}) e^{-j \omega T n}, \\
x(\boldsymbol{n}) & =\frac{1}{(2 \pi)^{D}} \int_{\boldsymbol{\omega} \in[-\pi, \pi)^{D}} X_{F}(\boldsymbol{\omega}) e^{j \boldsymbol{\omega}^{T} \boldsymbol{n} d \omega} .
\end{aligned}
$$

$z=\left[\begin{array}{llll}z_{0} & z_{1} & \cdots & z_{D-1}\end{array}\right]^{T}$ variable of the $z$ transform of MD signals. The $z$ transform of $x(n)$, where it converges, is given by

$$
X(z)=\sum_{n \in \mathfrak{T}} x(n) z^{-n}
$$

A vector raised to a vector power [2], as in $z^{-n}$ above, gives a scalar defined as $z^{n} \triangleq z_{0}^{n_{0}} z_{1}^{n_{1}} \cdots z_{D-1}^{n_{D-1}}$. Note that the subscript $F$ is used to distinguish the Fourier transform from the $z$ transform. It is clear that $\dot{X_{F}}(\omega)$ can be obtained by evaluating $X(z)$ at $z_{i}=e^{j \omega_{i}}$ for $i=$ $0, \cdots, D-1$.

Let $M$ be a $D \times D$ nonsingular integer matrix. Then, $\mathfrak{T}(\boldsymbol{M})$ is the set of all integer vectors of the form $\boldsymbol{M x}$, $x \in[0,1)^{D}$. And, $J(\boldsymbol{M}) \triangleq|\operatorname{det} \boldsymbol{M}|$ (absolute determinant), which is also equal to the number of elements in $\Re(M)$.

An integer matrix $\boldsymbol{U}$ is called unimodular if [det $\boldsymbol{U}]=$ \pm 1 , i.e., $J(\boldsymbol{U})=1$. Clearly, for unimodular $\boldsymbol{U}, \boldsymbol{U}^{-1}$ $=[$ adjugate of $\boldsymbol{U}] /[\operatorname{det} \boldsymbol{U}]$ is also an integer matrix, and is unimodular.

$\boldsymbol{A} \stackrel{R}{=} \boldsymbol{B}(\boldsymbol{A}$ is a right associate of $\boldsymbol{B}[14],[17])$ : This means that there exists a unimodular integer matrix $V$ such that $\boldsymbol{A}=\boldsymbol{B} \boldsymbol{V}$. Clearly, $\boldsymbol{A} \stackrel{R}{=} \boldsymbol{B}$ if and only if (iff) $\boldsymbol{B} \stackrel{R}{=} \boldsymbol{A}$. Similarly, $\boldsymbol{A}$ is a left associate of $\boldsymbol{B}$ (denoted as $A \stackrel{L}{\underline{L}}$ ) if $\boldsymbol{A}=\boldsymbol{U} \boldsymbol{B}$ for some unimodular integer matrix $U$.

Let $V$ be a $D \times D$ nonsingular real matrix. Then, LAT $(V)$ (the lattice generated by $V$ [27], [16], [3]) denotes the set of all vectors of the form $V \boldsymbol{n}, \boldsymbol{n} \in \mathfrak{T}$. It can be shown that LAT $(\boldsymbol{A})=\operatorname{LAT}(\boldsymbol{B})$ iff $\boldsymbol{A} \stackrel{R}{=} \boldsymbol{B}$. Also, $\operatorname{SPD}(\boldsymbol{V})$ (the symmetric parallelepiped generated by $\boldsymbol{V}$ ) is the set of all real vectors of the form $V \boldsymbol{x}, \boldsymbol{x} \in$ $[-1,1)^{D}$.

2) Decimation: The $\boldsymbol{M}$-fold decimated version of $x(\boldsymbol{n})$ is defined as $y(\boldsymbol{n})=x(\boldsymbol{M n})$, where $\boldsymbol{M}$ is a nonsingular 
integer matrix. The frequency domain relation is

$$
Y_{F}(\omega)=\frac{1}{J(\boldsymbol{M})} \sum_{k \in \mathfrak{Z}\left(M^{7}\right)} X_{F}\left(M^{-T}(\omega-2 \pi k)\right) .
$$

3) Expansion: Let $L$ be a nonsingular integer matrix. The $\boldsymbol{L}$-fold expanded version of $x(\boldsymbol{n})$ is defined as

$$
y(n)= \begin{cases}x\left(L^{-1} n\right) & n \in \operatorname{LAT}(L) \\ 0 & \text { otherwise. }\end{cases}
$$

The frequency domain relation is $Y_{F}(\omega)=X_{F}\left(\boldsymbol{L}^{T} \boldsymbol{\omega}\right)$. The corresponding $z$ domain relation is

$$
Y(z)=X\left(z^{L}\right) .
$$

The notation of a vector raised to a matrix power [2], as in $z^{L}$ above, is a $D \times 1$ vector defined as $z^{P} \triangleq\left[z^{p_{0}} z^{p_{1}}\right.$ $\left.\cdots z^{p_{D-1}}\right]^{T}$, where $\boldsymbol{p}_{i}$ is the $i$ th column of $\boldsymbol{P}$.

Remark: In this paper, $\boldsymbol{M}$ and $\boldsymbol{L}$ always denote $D \times D$ nonsingular integer matrices, with the above meanings.

4) To prevent aliasing due to $\boldsymbol{M}$-fold decimation (or suppress images owing to $\boldsymbol{M}$-fold expansion), a decimation (or interpolation) filter $H(z)$ is necessary. Typically, this filter has a parallelepiped passband in the region $\omega=$ $\pi \boldsymbol{M}^{-T} \boldsymbol{x}$, for $\boldsymbol{x} \in[-1,1)^{D}$. Using the notation of SPD, we can rewrite this as $\omega \in \operatorname{SPD}\left(\pi M^{-T}\right)$. Fig. 5 shows the typical passband for

$$
\boldsymbol{M}=\left[\begin{array}{rr}
1 & -1 \\
1 & 2
\end{array}\right] .
$$

Note that some other choices of passband regions are possible [28], [29].

5) Division Theorem for Integer Vectors: Every integer vector $\boldsymbol{n}$ can be expressed as $\boldsymbol{n}=\boldsymbol{k}+\boldsymbol{M} \boldsymbol{n}_{0}$, for some $\boldsymbol{k} \in \mathfrak{N}(\boldsymbol{M})$, and $\boldsymbol{n}_{0} \in \mathfrak{N}$. Moreover, $\boldsymbol{k}$ and $\boldsymbol{n}_{0}$ are unique for a given $n$. We denote this relation as $\boldsymbol{k}=n \bmod \boldsymbol{M}$, or $\boldsymbol{k}=((\boldsymbol{n}))_{M}$.

6) Polyphase Decomposition: The polyphase components of $x(\boldsymbol{n})$ with respect to a given $\boldsymbol{M}$ are defined as

$$
e_{k}(n)=x(M n+k), \quad \text { (Type 1) }
$$

or

$$
r_{\boldsymbol{k}}(\boldsymbol{n})=x(\boldsymbol{M n}-\boldsymbol{k}), \quad \text { (Type 2) }
$$

where $\boldsymbol{k} \in \mathscr{N}(\boldsymbol{M})$. So $\boldsymbol{k}$ can take on $J(\boldsymbol{M})$ different values. Also, in the frequency domain, the polyphase decomposition of $X_{F}(\omega)$ can be expressed as

$$
X_{F}(\boldsymbol{\omega})=\sum_{k \in \mathfrak{N}(M)} e^{-j \boldsymbol{\omega}^{T} k} E_{F, k}\left(M^{T} \boldsymbol{\omega}\right),
$$

or

$$
X_{F}(\omega)=\sum_{k \in \mathcal{N}(M)} e^{j \omega^{T} k} R_{F, k}\left(M^{T} \omega\right) .
$$

In the $z$ domain, these become

$$
X(z)=\sum_{k \in \mathscr{N}(M)} z^{-k} E\left(z^{M}\right),
$$

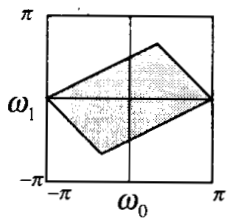

Fig. 5. Typical passband of multirate filters.

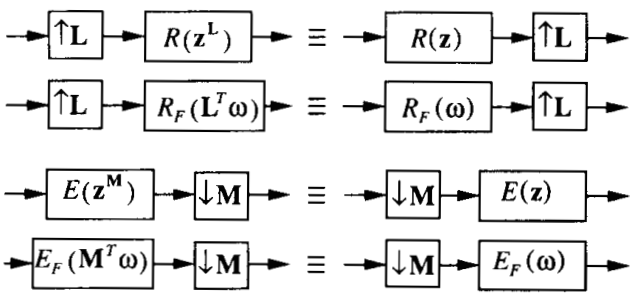

Fig. 6. MD Noble identities.

or

$$
X(z)=\sum_{k \in \Re(M)} z^{k} R\left(z^{M}\right) . \quad \text { (Type 2) }
$$

Note that $z^{k}$ (or $e^{j \omega^{T} k}$ ) is a shift operator, which shifts (advances) an MD signal by a vector amount $\boldsymbol{k}$. Similarly, $z^{-k}\left(\right.$ or $\left.e^{-j \omega t}\right)$ shifts an MD signal by $-k$, i.e., "delays", it by $\boldsymbol{k}$.

7) Noble Identities: These are rules which permit us to move decimators and expanders across transfer functions. For example, a filter followed by a $\boldsymbol{L}$-fold expander is equivalent to the expander followed by the $\boldsymbol{L}$-fold expanded version of the same filter. Also, a $\boldsymbol{M}$-fold decimator followed by a filter is equivalent to the $\boldsymbol{M}$-fold expanded version of the same filter followed by the decimator. Fig. 6 shows these rules.

8) Periodicity Matrix: An MD signal $x(n)$ is said to be periodic with periodicity matrix $\boldsymbol{L}$ if $x(\boldsymbol{n}+\boldsymbol{L k})=x(\boldsymbol{n})$, $\forall k \in \mathfrak{N}$. Further details can be found in Section VI.

\section{GCRD, GCLD, LCRM, AND LCLM}

In this section, we introduce some properties about integer matrices. As mentioned before, the counterpart of these properties in polynomial matrix case is well known and well developed [30], [18], [17]. In the [17, appendix], many of these properties are also given for matrices with elements in a principle ideal domain (pid), which is an even more general case. We omit proofs which can be found in these references. We will review the concepts of the $1 \mathrm{crm}$ and $\mathrm{Iclm}$ for the matrix case [14] and derive several properties, including their relations with gcrd/gcld. We shall also present a method of finding an $1 \mathrm{crm} / \mathrm{lclm}$ of two integer matrices. All of these will be applied in deriving many of the new multirate results in the following sections. 
A. Gcrd and Gcld [14], [18], [17]

Definitions:

1) The integer matrix $\boldsymbol{R}$ is a right divisor ( $\mathrm{rd}$ ) of $\boldsymbol{M}$ if $\boldsymbol{M}=\boldsymbol{P} \boldsymbol{R}$ for some integer matrix $\boldsymbol{P}$.

2) The integer matrix $\boldsymbol{R}$ is a common right divisor of $M$ and $L$ (denoted by $\operatorname{crd}(M, L)$ ) if $M=P R$ and $L=Q R$ for some integer matrices $P$ and $\boldsymbol{Q}$.

3) The integer matrix $\boldsymbol{R}_{0}$ is a greatest common right divisor of $\boldsymbol{M}$ and $\boldsymbol{L}$ (denoted by $\operatorname{gcrd}(\boldsymbol{M}, \boldsymbol{L})$ ) if

i) $R_{0}$ is a crd $(M, L)$.

ii) If $\boldsymbol{R}$ is another crd $(\boldsymbol{M}, \boldsymbol{L}), \boldsymbol{R}_{0}=\boldsymbol{S R}$ for some integer matrix $S$.

4) Two matrices $\boldsymbol{M}$ and $\boldsymbol{L}$ are right coprime if all their crd's are unimodular.

Properties:

1) The gerd is not unique. In fact, all gerd's of two given integer matrices are left associate of one another. Hence, we should write " $\boldsymbol{R}_{0} \stackrel{\underline{L}}{=} \operatorname{gcrd}(\boldsymbol{M}, \boldsymbol{L})$ " (instead of " $\boldsymbol{R}_{0}=\operatorname{gcrd}(M, L)$ ") to express " $\boldsymbol{R}_{0}$ is a gcrd of $\boldsymbol{M}$ and L."

2) Given $\boldsymbol{M}=\boldsymbol{P R}$ and $\boldsymbol{L}=\boldsymbol{Q R}$. Then, $\boldsymbol{R}$ is a gcrd ( $\boldsymbol{M}$, $L)$ iff $\boldsymbol{P}$ and $\boldsymbol{Q}$ are right coprime.

3) Let $\boldsymbol{R}_{0}$ be a gcrd $(\boldsymbol{M}, \boldsymbol{L})$. There exist integer matrices $\boldsymbol{A}$ and $\boldsymbol{B}$ such that $\boldsymbol{A} \boldsymbol{M}+\boldsymbol{B} \boldsymbol{L}=\boldsymbol{R}_{0}$. This is the extension of the Euclid's theorem.

4) Suppose $\boldsymbol{M}$ and $\boldsymbol{L}$ are right coprime. There exist integer matrices $\boldsymbol{A}$ and $\boldsymbol{B}$ such that $\boldsymbol{A M}+\boldsymbol{B} \boldsymbol{L}=\boldsymbol{I}$. This is called the generalized Bezout theorem.

Remarks:

1) The left divisor (ld), common left divisor (cld), greatest common left divisor (gcld) and left coprimeness are defined similarly.

2) The proof of the existence of gcrd/gcld and methods for finding $\mathrm{gcrd} / \mathrm{gcld}$ can be found in [14], [18], [17].

\section{B. Smith Form and Smith-McMillan Form [30], [18],} [17]

1) Smith Form: Any nonsingular integer matrix $\boldsymbol{M}$ can always be decomposed as $\boldsymbol{M}=\boldsymbol{U} \boldsymbol{\Lambda} \boldsymbol{V}$ where $\boldsymbol{U}$ and $\boldsymbol{V}$ are unimodular integer matrices and $\boldsymbol{\Lambda}$ is a diagonal matrix with nonzero integer elements on the diagonal.

Remarks: The Smith form has been used outside the control-theory literature more than once. For example, the Smith form for polynomial matrices with coefficients in a finite field has been mentioned and applied in convolutional coding theory in [15]. A special Smith form was used to design MD filter banks in [6]. The Smith form for integer matrices has been used in [25, problem 2.20] and [31] for computing the MD discrete Fourier transform, and was used in the MD multirate systems to exploit the decimation/expansion matrices in [9], [10].

2) Smith-McMillan Form: Any nonsingular matrix $\boldsymbol{H}$ with rational elements can always be decomposed as $\boldsymbol{H}=$ $\boldsymbol{U} \boldsymbol{\Lambda} \boldsymbol{V}$ where $\boldsymbol{U}$ and $\boldsymbol{V}$ are unimodular integer matrices and
$\boldsymbol{\Lambda}$ is a diagonal matrix with nonzero rational elements on the diagonal.

\section{Right/Left Matrix Fraction Description (MFD)}

Any nonsingular matrix $\boldsymbol{H}$ with rational elements can be expressed as $\boldsymbol{H}=\boldsymbol{P}_{1} \boldsymbol{Q}_{1}^{-1}$ (right MFD), or as $\boldsymbol{H}=$ $\boldsymbol{Q}_{2}^{-1} \boldsymbol{P}_{2}$ (left MFD), where $\boldsymbol{P}_{i}$ 's and $\boldsymbol{Q}_{i}$ 's are nonsingular integer matrices. A right MFD is said to be irreducible if $\boldsymbol{P}_{1}$ and $\boldsymbol{Q}_{1}$ are right coprime. Similarly, a left MFD is said to be irreducible if $\boldsymbol{P}_{2}$ and $Q_{2}$ are left coprime [18], [17].

It can be shown that if $\boldsymbol{P}_{1} \boldsymbol{Q}_{1}^{-1}$ and $\boldsymbol{P}_{1}^{\prime} \boldsymbol{Q}_{1}^{\prime-1}$ are both irreducible right MFD's of $\boldsymbol{H}$, then there exist unimodular $\boldsymbol{V}$ such that $\boldsymbol{P}_{1}^{\prime}=\boldsymbol{P}_{1} \boldsymbol{V}$ and $\boldsymbol{Q}_{1}^{\prime}=\boldsymbol{Q}_{1} \boldsymbol{V}$, i.e., $\boldsymbol{P}_{1}^{\prime}$ and $\boldsymbol{Q}_{1}^{\prime}$ are right associates of $\boldsymbol{P}_{1}$ and $\boldsymbol{Q}_{1}$ with the same $\boldsymbol{V}$. Therefore, all the denominator matrices (the $Q$ 's) of the irreducible right MFD's have the same absolute determinant $d_{1}$. Similarly, we can show that all the denominator matrices of the irreducible left MFD's have the same absolute determinant $d_{2}$. These, in turn, are equal, i.e., $d_{1}=$ $d_{2}$, as explained later.

Computation of Irreducible MFD's Using the SmithMcMillan Form [17]: For a given rational matrix $\boldsymbol{H}$, first decompose it into Smith-McMillan form, $\boldsymbol{H}=\boldsymbol{U} \boldsymbol{\Lambda} \boldsymbol{V}$, where $\boldsymbol{\Lambda}=\operatorname{diag}\left[\lambda_{0}, \cdots, \lambda_{D-1}\right]$. Then, represent all the rational $\lambda_{i}=\alpha_{i} / \beta_{i}$ in their irreducible forms $\left(\alpha_{i}\right.$ and $\beta_{i}$ are coprime integers for all $i$ ). Let $\boldsymbol{\Lambda}_{\alpha}=\operatorname{diag}\left[\alpha_{0}, \cdots\right.$, $\left.\alpha_{D-1}\right]$ and $\boldsymbol{\Lambda}_{\beta}=\operatorname{diag}\left[\beta_{0}, \cdots, \beta_{D-1}\right]$ so that

$$
\boldsymbol{H}=\underbrace{\boldsymbol{U} \boldsymbol{\Lambda}_{\alpha}}_{\boldsymbol{P}_{1}} \underbrace{\boldsymbol{\Lambda}_{\beta}^{-1} \boldsymbol{V}}_{\boldsymbol{Q}_{1}^{-1}}=\underbrace{\boldsymbol{U} \boldsymbol{\Lambda}_{\beta}^{-1}}_{\boldsymbol{Q}_{2}^{-1}} \underbrace{\boldsymbol{\Lambda}_{\alpha} \boldsymbol{V}}_{\boldsymbol{P}_{2}} .
$$

It can be shown that $\boldsymbol{P}_{1}$ and $\boldsymbol{Q}_{1}$ are right coprime and $\boldsymbol{P}_{2}$ and $Q_{2}$ are left coprime, so $\boldsymbol{P}_{1} \boldsymbol{Q}_{1}^{-1}$ and $\boldsymbol{Q}_{2}^{-1} \boldsymbol{P}_{2}$ are irreducible MFD's.

Note that (3.1) tells us that there exist one irreducible right MFD and one irreducible left MFD of which the denominator matrices have the same absolute determinant, since $J\left(\boldsymbol{Q}_{1}\right)=J\left(\boldsymbol{\Lambda}_{\beta}\right)=J\left(\boldsymbol{Q}_{2}\right)$. Summarizing, we have proved the following:

Fact 1: For all irreducible right and left MFD's of a nonsingular rational matrix, the denominator matrices have the same absolute determinant.

\section{Lcrm and Lclm \\ Definitions:}

1) $\boldsymbol{R}$ is a right multiple ( $\mathrm{rm}$ ) of $\boldsymbol{M}$ if $\boldsymbol{R}=\boldsymbol{M P}$ for some integer matrix $\boldsymbol{P}$, i.e., if $\boldsymbol{M}$ is a left divisor of $\boldsymbol{R}$.

2) $\boldsymbol{R}$ is a common right multiple of $\boldsymbol{M}$ and $\boldsymbol{L}$ (denoted as crm $(\boldsymbol{M}, \boldsymbol{L})$ ) if $\boldsymbol{R}=\boldsymbol{M P}=\boldsymbol{L} \boldsymbol{Q}$ for some integer matrices $\boldsymbol{P}$ and $\boldsymbol{Q}$.

3) $\boldsymbol{R}_{0}$ is a least common right multiple of $\boldsymbol{M}$ and $L$ (denoted as $\operatorname{lcrm}(\boldsymbol{M}, \boldsymbol{L}))$ if

i) $R_{0}$ is a nonsingular crm $(M, L)$.

ii) If $\boldsymbol{R}$ is another nonsingular crm $(\boldsymbol{M}, \boldsymbol{L})$, then $\boldsymbol{R}=$ $\boldsymbol{R}_{0} \boldsymbol{S}$ for some integer matrix $\boldsymbol{S}$. 
Remark on Singularity: Singular crm is of less importance because given any nonsingular $\operatorname{crm}(\boldsymbol{M}, \boldsymbol{L})$, we can always postmultiply it by a singular matrix to get a singular crm $(\boldsymbol{M}, \boldsymbol{L})$. Also, if either $\boldsymbol{M}$ or $\boldsymbol{L}$ is singular, all $\mathrm{crm}(\boldsymbol{M}, \boldsymbol{L})$ 's are singular and it is meaningless to discuss the $\mathrm{lcrm}(\boldsymbol{M}, \boldsymbol{L})$. For these various reasons, by definition we restrict the lcrm to be nonsingular and to be defined only for nonsingular $\boldsymbol{M}$ and $\boldsymbol{L}$. This is slightly different from the definition in [14], but more proper for our discussions. This is also consistent with the convention for the $1 \mathrm{D}$ case, where we exclude zero as a least common multiple although it is a multiple of any integer.

Note that $\mathrm{crm}(\boldsymbol{M}, \boldsymbol{L})$ and $1 \mathrm{crm}(\boldsymbol{M}, \boldsymbol{L})$ are not unique. According to the above definitions, we can prove the following:

Lemma 1:

a) If $\boldsymbol{A}$ and $\boldsymbol{B}$ are both lcrm $(\boldsymbol{M}, L)$, then $\boldsymbol{A} \stackrel{R}{=} \boldsymbol{B}$ [14].

b) Let $\boldsymbol{B}$ be an lcrm $(\boldsymbol{M}, \boldsymbol{L})$. Then, $\boldsymbol{A}$ is also an lcrm $(\boldsymbol{M}, \boldsymbol{L})$ iff $\boldsymbol{A} \stackrel{R}{=} B$.

\section{Proofs:}

a) According to the definition of Icrm, we have $A=$ $B S$ and $B=A T$. Then, $A=B S=A T S$, so $T S=I$. This implies both $\boldsymbol{S}$ and $\boldsymbol{T}$ are unimodular, i.e., $\boldsymbol{A} \stackrel{R}{=} \boldsymbol{B}$.

b) The "only if" part follows directly from a). We proceed to prove the "if", part. Suppose $\boldsymbol{A}=\boldsymbol{B} \boldsymbol{U}$, where $\boldsymbol{U}$ is unimodular. Clearly, $\boldsymbol{A}$ is a crm of $\boldsymbol{M}$ and $\boldsymbol{L}$. Since $\boldsymbol{B}$ is an Icrm of $\boldsymbol{M}$ and $\boldsymbol{L}$, any crm of $\boldsymbol{M}$ and $\boldsymbol{L}$, say $\boldsymbol{R}$, can be written as $\boldsymbol{R}=\boldsymbol{B S}$. So, we have

$$
R=\underbrace{B U}_{A} \underbrace{U^{-1} S}_{S^{\prime}}
$$

where $S^{\prime}$ is also an integer matrix. This proves that $\boldsymbol{A}$ is an Icrm.

Remarks:

$\triangle \triangle \triangle$

1) It can be easily verified that there exists at least one crm $(\boldsymbol{M}, \boldsymbol{L})$, which is $\boldsymbol{R}=\boldsymbol{M}( \pm$ adjugate of $\boldsymbol{M}] \boldsymbol{L})=$ $\boldsymbol{L}(J(\boldsymbol{M}) \boldsymbol{I})$. On the other hand, the existence of a nonsingular lcrm of any two nonsingular matrices is guaranteed by a constructive method (Section III-E).

2) From the above properties, it also can be easily verified that an $\mathrm{lcrm}$ is a nonsingular crm with the smallest absolute determinant. And, all the lcrm $(\boldsymbol{M}, \boldsymbol{L})$ 's have the same absolute determinant. In particular, Lemma 1 says that the $\operatorname{Icrm}(\boldsymbol{M}, \boldsymbol{L})$ is unique up to postmultiplication by a unimodular matrix. Therefore, for consistency in notation, we should write " $\boldsymbol{R}_{0} \stackrel{R}{=} \operatorname{lcrm}(\boldsymbol{M}, \boldsymbol{L})$ " (instead of " $\boldsymbol{R}_{0}=\operatorname{lcrm}(\boldsymbol{M}, \boldsymbol{L})$ ') to express " $\boldsymbol{R}_{0}$ is an Icrm of $\boldsymbol{M}$ and L.",

Next, we can relate lcrm together with gcrd and right coprimeness by the following theorem:

Theorem 1: Let $\boldsymbol{R}$ be a nonsingular $\mathrm{crm}(\boldsymbol{M}, \boldsymbol{L})$, i.e., $\boldsymbol{R}=\boldsymbol{M P}=\boldsymbol{L} \boldsymbol{Q}$. Then, $\boldsymbol{R}$ is an $\operatorname{lcrm}(\boldsymbol{M}, \boldsymbol{L})$ iff $\boldsymbol{P}$ and $\boldsymbol{Q}$ are right coprime.
Proof:

1) If $\boldsymbol{P}$ and $\boldsymbol{Q}$ are not right coprime, then there exists an $\boldsymbol{X}$ which is not unimodular such that $\boldsymbol{P}=\boldsymbol{P}^{\prime} \boldsymbol{X}$ and $\boldsymbol{Q}$ $=\boldsymbol{Q}^{\prime} \boldsymbol{X}$. Therefore, we have

$$
R=\underbrace{M P^{\prime}}_{R^{\prime}} X=\underbrace{L Q^{\prime} X}_{R^{\prime}}
$$

Clearly, $R=R^{\prime} \boldsymbol{X}$, and $\boldsymbol{R}^{\prime}$ is a crm of $\boldsymbol{M}$ and $\boldsymbol{L}$. Suppose $\boldsymbol{R}$ is an lcrm of $\boldsymbol{M}$ and $\boldsymbol{L}$, then $\boldsymbol{R}^{\prime}=\boldsymbol{R S}$ according to the definition of lcrm. Then, $\boldsymbol{R}=\boldsymbol{R}^{\prime} \boldsymbol{X}=\boldsymbol{R} \boldsymbol{S} \boldsymbol{X}$, which implies both $\boldsymbol{X}$ and $\boldsymbol{S}$ must be unimodular and leads to contradiction. Hence we conclude that if $\boldsymbol{P}$ and $\boldsymbol{Q}$ are not right coprime, $\boldsymbol{R}$ is not an Icrm of $\boldsymbol{M}$ and $\boldsymbol{L}$.

2) Next, suppose $\boldsymbol{P}$ and $\boldsymbol{Q}$ are right coprime, we have to prove that $\boldsymbol{R}$ is an lcrm of $\boldsymbol{M}$ and $\boldsymbol{L}$. Let $\boldsymbol{R}^{\prime}$ be any other nonsingular crm of $M$ and $L$, i.e., $R^{\prime}=M P^{\prime}=L Q^{\prime}$. Clearly, $\boldsymbol{P}^{\prime}$ is nonsingular. Because $\boldsymbol{P}$ and $\boldsymbol{Q}$ are right coprime, there exist integer matrices $\boldsymbol{A}$ and $\boldsymbol{B}$ such that $\boldsymbol{A P}$ $+\boldsymbol{B} \boldsymbol{Q}=\boldsymbol{I}$ (generalized Bezout theorem). Replacing $\boldsymbol{Q}$ with $\boldsymbol{Q}^{\prime} \boldsymbol{P}^{\prime-1} \boldsymbol{P}$, we can rewrite this as

$$
\boldsymbol{A} \boldsymbol{P}^{\prime} \boldsymbol{P}^{\prime-1} \boldsymbol{P}+\boldsymbol{B} \boldsymbol{Q}^{\prime} \boldsymbol{P}^{\prime-1} \boldsymbol{P}=\boldsymbol{I} .
$$

Postmultiplying both sides by $\boldsymbol{P}^{-1} \boldsymbol{P}^{\prime}$, we get

$$
\underbrace{A P^{\prime}+B Q^{\prime}}_{S}=P^{-1} P^{\prime} .
$$

So, $\boldsymbol{P}^{\prime}=\boldsymbol{P S}$ and hence $\boldsymbol{R}^{\prime}=\boldsymbol{R S}$. From the definition of lcrm, $\boldsymbol{R}$ is indeed an $\operatorname{lcrm}(\boldsymbol{M}, \boldsymbol{L})$. $\triangle \Delta \triangle$

Remark: The left multiple (lm), common left multiple (clm), and least common left multiple (lclm) can be defined similarly. All the properties above can also be derived similarly.

\section{E. Computation of Lcrm/Lclm Using MFD's}

A method of computing an $\mathrm{lcrm} / \mathrm{lclm}$ of two nonsingular matrices can be found in $[14$, p. 36]. However, the above-mentioned irreducible MFD's give us an alternative. This also gives a constructive way of proving the existence of a nonsingular $\mathrm{lcrm} / \mathrm{lclm}$ of two nonsingular matrices.

To compute an lcrm of nonsingular $\boldsymbol{M}$ and $\boldsymbol{L}$, we let $\boldsymbol{H}$ $=\boldsymbol{M}^{-1} \boldsymbol{L}$ (which is also nonsingular) and compute one irreducible right MFD of $\boldsymbol{H}$ as in (3.1), so we have $\boldsymbol{M}^{-1} \boldsymbol{L}$ $=P_{1} Q_{1}^{-1}$ where $P_{1}$ and $Q_{1}$ are right coprime. Therefore, $\boldsymbol{M} \boldsymbol{P}_{1}=\boldsymbol{L} \boldsymbol{Q}_{1}$. Denote this as $\boldsymbol{R}$. Using Theorem 1 , we can conclude that $\boldsymbol{R}$ is an $\operatorname{lcrm}(\boldsymbol{M}, \boldsymbol{L})$. Similarly, if we let $\boldsymbol{H}^{\prime}$ $=\boldsymbol{L} \boldsymbol{M}^{-1}$ and compute one irreducible left MFD of it using (3.1), then $\boldsymbol{R}^{\prime}=\boldsymbol{P}_{2} \boldsymbol{M}=\boldsymbol{Q}_{2} \boldsymbol{L}$ is an $\operatorname{lclm}(\boldsymbol{M}, \boldsymbol{L})$.

\section{Polyphase Implementations of Rational SAMPling Rate Alterations}

A 1D sampling rate alteration system with decimation ratio $M / L$ can be implemented efficiently by using the rational polyphase implementation (RPI) as in Fig. 3(d). 
For this technique to work, $M$ and $L$ should be coprime. In this section, we shall extend this technique to the MD case, which finds applications in conversions of images or video data between different sampling standards. An MD decimation system with rational decimation ratio (in this case, a matrix) $\boldsymbol{H}=\boldsymbol{L}^{-1} \boldsymbol{M}$ is shown in Fig. 7. As an example, if we choose

$$
\boldsymbol{L}=\left[\begin{array}{ll}
2 & 0 \\
0 & 1
\end{array}\right] \quad \text { and } \quad \boldsymbol{M}=\left[\begin{array}{rr}
1 & 1 \\
1 & -1
\end{array}\right]
$$

we can convert rectangularly sampled images to hexagonally sampled ones, as shown in [19, fig. 6]. As in the $1 \mathrm{D}$ case, the MD filter $H(z)$ in Fig. 7 is used to suppress image components generated by the $L$-fold expander and eliminate aliasing owing to the $\boldsymbol{M}$-fold decimator.

We can use the MD polyphase decomposition to implement this system more efficiently as in either Fig. 8(a) or (b). The numbers of branches in Figs. 8(a) and (b) are $J(\boldsymbol{M})$ and $J(L)$, respectively. For simplicity, figures only show the case where $J(\boldsymbol{M})=3$ and $J(\boldsymbol{L})=2$. We can use the RPI technique to improve the efficiency even further. Fig. 9 shows this by successively redrawing the circuit of Fig. 8(a).

Starting from Fig. 8(a), suppose it is possible to replace every $\boldsymbol{k}_{i}$ in $\mathfrak{N}(\boldsymbol{M})$ with $\boldsymbol{M} \boldsymbol{k}_{i 1}+\boldsymbol{L k}_{i 2}$, where $\boldsymbol{k}_{i 1}$ and $\boldsymbol{k}_{i 2}$ are some integer vectors. Hence we get Fig. 9(a). With the help of Noble identities, Fig. 9(a) can be redrawn as Fig. 9(b). Next, suppose we can interchange the expanders and decimators to obtain Fig. 9(c). We can then perform Type 2 polyphase decomposition on $E_{k}(z)$ 's with respect to $L$, and get Fig. 9(d). In summary, Fig. 9(d) is equivalent to Fig. 8(a) but each arithmetic operation is now performed at its lowest rate. Note that the filters $E_{k_{j}}(z)$ in Fig. 9(d) are the $\boldsymbol{M L}$-fold polyphase components (up to a certain delay) of $H(z)$.

To summarize, we can see that the following two issues should be considered for the above technique to work: 1) Every $\boldsymbol{k}_{\boldsymbol{i}}$ in $\mathfrak{K}(\boldsymbol{M})$ should be expressed in the form of $\boldsymbol{k}_{i}$ $=\boldsymbol{M} \boldsymbol{k}_{i 1}+\boldsymbol{L} \boldsymbol{k}_{i 2}$, where $\boldsymbol{k}_{i 1}$ and $\boldsymbol{k}_{i 2}$ are some integer vectors. 2) The decimators and the expanders should be interchangeable.

We shall devote the rest of this section to the proof of the following simple and clear statement.

Theorem 2: The above two issues are satisfied if and only if 1) $\boldsymbol{M L}=\boldsymbol{L} \boldsymbol{M}$, i.e., $\boldsymbol{M}$ and $\boldsymbol{L}$ commute. 2) $\boldsymbol{M}$ and $\boldsymbol{L}$ are coprime. (As we will show, left coprimeness is equivalent to right coprimeness when $\boldsymbol{M L}=\boldsymbol{L M}$.)

We first deal with the interchangeability of decimators and expanders and prove the following theorem.

Theorem 3: The $\boldsymbol{L}$-fold expander and the $\boldsymbol{M}$-fold decimator can be interchanged if and only if 1) $\boldsymbol{M L}=\boldsymbol{L M}$. 2) $\boldsymbol{M L}$ is an $\operatorname{lcrm}(\boldsymbol{M}, \boldsymbol{L})$, i.e., $\boldsymbol{M L} \stackrel{R}{=} \operatorname{lcrm}(\boldsymbol{M}, \boldsymbol{L})$. (Given $\boldsymbol{M L}=\boldsymbol{L} \boldsymbol{M}$, this condition can be shown to be equivalent to the coprimeness of $\boldsymbol{M}$ and $\boldsymbol{L}$. See Theorem 4.)

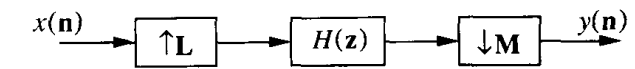

Fig. 7. MD rational decimation system.

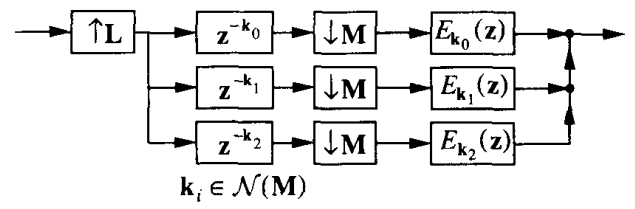

(a)

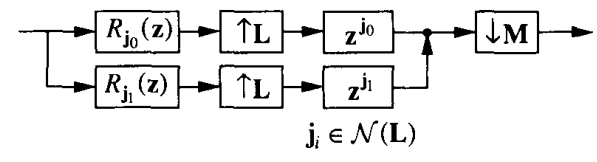

(b)

Fig. 8. Two types of polyphase implementations of an MD rational decimation system.

\section{Comments:}

1) These two conditions can be easily tested. While the test for " $\boldsymbol{M L}=\boldsymbol{L} \boldsymbol{M}$ ', is straight-forward, the test for coprimeness (i.e., the computation of a gcrd/gcld) is also easy and can be found in [14], [18], [17].

2) In the $1 \mathrm{D}$ case, Condition 1 is automatic, and Condition 2 is equivalent to coprimeness.

Proof: Consider $y_{1}(\boldsymbol{n})$ and $y_{2}(\boldsymbol{n})$ in Fig. 10. From the definitions of $\boldsymbol{M}$-fold decimation and $\boldsymbol{L}$-fold expansion, we have

$$
y_{1}(\boldsymbol{n})= \begin{cases}x\left(M L^{-1} n\right) & n \in \operatorname{LAT}(\boldsymbol{L}) \\ 0 & \text { otherwise }\end{cases}
$$

and

$$
y_{2}(\boldsymbol{n})= \begin{cases}x\left(\boldsymbol{L}^{-1} \boldsymbol{M n}\right) & \boldsymbol{M n} \in \mathrm{LAT}(\boldsymbol{L}) \\ 0 & \text { otherwise. }\end{cases}
$$

For $y_{1}(n)$ to be identical to $y_{2}(n)$, we should in particular have $x\left(M L^{-1} n\right)=x\left(\boldsymbol{L}^{-1} \boldsymbol{M n}\right)$ for $n \in$ LAT $(\boldsymbol{L})$. Because $x(n)$ is arbitrary, we should have

$$
\boldsymbol{M} \boldsymbol{L}^{-1} \boldsymbol{n}=\boldsymbol{L}^{-1} \boldsymbol{M n} \quad \forall \boldsymbol{n} \in \operatorname{LAT}(\boldsymbol{L}) .
$$

Since $L$ is not singular, LAT $(\boldsymbol{L})$ contains $D$ linearly independent vectors. So, the above implies $\boldsymbol{M} \boldsymbol{L}^{-1}=\boldsymbol{L}^{-1} \boldsymbol{M}$, or, $\boldsymbol{L} \boldsymbol{M}=\boldsymbol{M L}$

In order for $y_{1}(\boldsymbol{n})=y_{2}(\boldsymbol{n})$, we also need

$$
\boldsymbol{n} \in \operatorname{LAT}(\boldsymbol{L}) \quad \text { iff } \quad \boldsymbol{M n} \in \operatorname{LAT}(\boldsymbol{L}) .
$$

Since $\boldsymbol{M n}$ is also in LAT $(\boldsymbol{M})$, we know $\boldsymbol{M n} \in \operatorname{LAT}(\boldsymbol{L})$ iff $\boldsymbol{M n} \in \operatorname{LAT}(\boldsymbol{L}) \cap \operatorname{LAT}(\boldsymbol{M})$. It is shown in the Appendix that $\operatorname{LAT}(\boldsymbol{L}) \cap \operatorname{LAT}(\boldsymbol{M})=\operatorname{LAT}(\operatorname{lcrm}(\boldsymbol{M}, \boldsymbol{L}))$. Let $\boldsymbol{M P}$ be an lcrm $(\boldsymbol{M}, \boldsymbol{L})$. Then, the right-hand side of (4.4) is equivalent to $\boldsymbol{M n} \in \mathrm{LAT}(\boldsymbol{M P})$, which is true iff $\boldsymbol{n} \in \mathrm{LAT}$ 

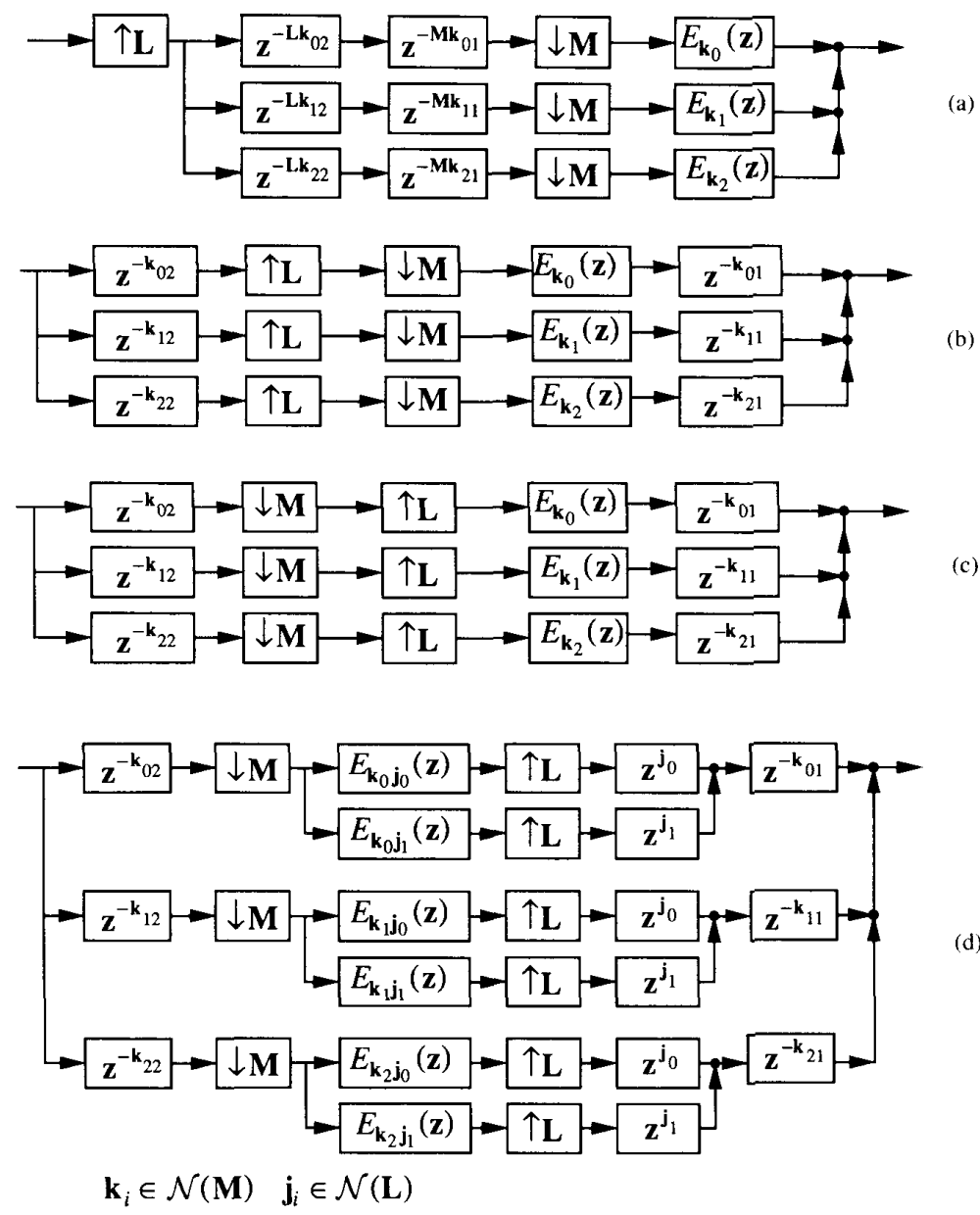

Fig. 9. Successive redrawing of polyphase implementations of an MD rational decimation system.

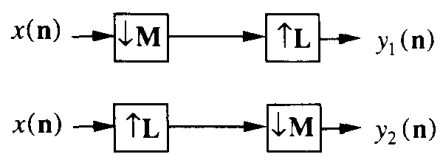

Fig. 10. Interchange of an MD decimator and an MD expander.

$(\boldsymbol{P})$. Comparing this with the left-hand side of (4.4), we know that for (4.4) to be true, $L$ and $\boldsymbol{P}$ should generate the same lattice, i.e., $\boldsymbol{L} \stackrel{R}{=} \boldsymbol{P}$. Then, $\boldsymbol{M L} \stackrel{R}{=} \boldsymbol{M P}$ and hence $M L$ should be an lcrm $(M, L)$.

$\triangle \triangle \triangle$

Remark: The interchangeability problem was also addressed in [1] for the 2D case for upper triangular $M$ and $L$. However, the conditions we proved above work for any $\boldsymbol{M}, \boldsymbol{L}$ and any number of dimensions. In fact, the result in [1] is a special case of the results presented here.

It turns out that when $\boldsymbol{M L}=\boldsymbol{L M}$, the $\mathrm{lcrm} / \mathrm{lclm}$ and gcrd/gcld (or right/left coprimeness) have very strong relations, as stated in the following theorem.
Theorem 4: When $\boldsymbol{M L}=\boldsymbol{L M}$, the following four statements are equivalent:

1) $M L$ is an $\operatorname{lcrm}(M, L)$.

2) $\boldsymbol{M}$ and $\boldsymbol{L}$ are right coprime.

3) $M$ and $L$ are left coprime.

4) $\boldsymbol{M} L$ is an $\operatorname{lclm}(\boldsymbol{M}, \boldsymbol{L})$.

For the 1D case, this theorem simply states " $1 \mathrm{~cm}(M$, $L$ ) $=M L$ iff $M$ and $L$ are coprime," a well-known fact.

Proof: Let $\boldsymbol{R}=\boldsymbol{M L}=\boldsymbol{L} \boldsymbol{M}$. Using Theorem 1, we know that $\boldsymbol{R}$ is an lcrm $(\boldsymbol{M}, \boldsymbol{L})$ iff $\boldsymbol{L}$ and $\boldsymbol{M}$ are right coprime. That is, Statements 1 and 2 imply each other. Similarly, we can show that Statements 3 and 4 imply each other.

Next, consider Statements 2 and 3. Let $\boldsymbol{H}=\boldsymbol{M L ^ { - 1 }}=$ $\boldsymbol{L}^{-1} \boldsymbol{M}$. If $\boldsymbol{M}$ and $\boldsymbol{L}$ are right coprime, $\boldsymbol{M} \boldsymbol{L}^{-1}$ is an irreducible right MFD of $\boldsymbol{H}$. Suppose $\boldsymbol{M}$ and $\boldsymbol{L}$ are not left coprime. Let $\boldsymbol{X}$ (nonunimodular) be a gcld of $\boldsymbol{M}$ and $\boldsymbol{L}$, i.e., $\boldsymbol{M}=\boldsymbol{X} \boldsymbol{M}^{\prime}, \boldsymbol{L}=\boldsymbol{X} \boldsymbol{L}^{\prime}$ where $\boldsymbol{M}^{\prime}$ and $\boldsymbol{L}^{\prime}$ are left coprime. Then, $\boldsymbol{H}=\boldsymbol{L}^{\prime-1} \boldsymbol{M}^{\prime}$ is an irreducible left MFD. 
We then have $J\left(\boldsymbol{L}^{\prime}\right)<J(\boldsymbol{L})$, which violates Fact I (Section III-C). Hence, we conclude that Statement 2 implies Statement 3. Similarly, we can prove that Statement $3 \mathrm{im}-$ plies Statement 2 and this completes the proof. $\triangle \triangle \triangle$

Next, we shall consider the feasibility of expressing every $\boldsymbol{k}_{i}$ in $\mathfrak{X}(\boldsymbol{M})$ in the form of $\boldsymbol{k}_{i}=\boldsymbol{M} \boldsymbol{k}_{i 1}+\boldsymbol{L} \boldsymbol{k}_{i 2}$.

Lemma 2: If $\boldsymbol{M}$ and $\boldsymbol{L}$ are left coprime, then any integer vector $\boldsymbol{k}$ can be expressed as $\boldsymbol{k}=\boldsymbol{M} \boldsymbol{k}_{1}+\boldsymbol{L} \boldsymbol{k}_{2}$ for some $\boldsymbol{k}_{1}$ and $\boldsymbol{k}_{2} \in \mathfrak{K}$.

Proof: If $\boldsymbol{M}$ and $\boldsymbol{L}$ are left coprime, there exist $\boldsymbol{P}$ and $Q$ such that $M P+L Q=I$ (generalized Bezout theorem). Then, for any $\boldsymbol{k} \in \mathfrak{N}$,

$$
M \underbrace{P k}_{k_{1}}+L \underbrace{Q k}_{k_{2}}=k
$$

$\triangle \triangle \triangle$

Combining Theorems 3, 4, and Lemma 2, we thus complete the proof of Theorem 2 . We conclude this section with the following result, which is intuitively appealing.

Lemma 3: Suppose $\boldsymbol{M L}=\boldsymbol{L} \boldsymbol{M}$. Then, any $\operatorname{lcrm}(\boldsymbol{M}, \boldsymbol{L})$ and any $\operatorname{gerd}(\boldsymbol{M}, \boldsymbol{L})$ can be related as $\operatorname{lcrm}(\boldsymbol{M}, \boldsymbol{L}) \cdot \boldsymbol{U}$. $\operatorname{gcrd}(\boldsymbol{M}, \boldsymbol{L})=\boldsymbol{M L}$ for some unimodular $\boldsymbol{U}$.

Remark: For the 1D case, this nicely reduces to $\mathrm{lcm}$ $(M, L) \operatorname{gcd}(M, L)=M L$. This lemma also holds for the $\operatorname{lclm}$ and gcld case, i.e., gcld $(\boldsymbol{M}, L) \cdot U \cdot \operatorname{lclm}(M, L)$ $=\boldsymbol{M L}$ for some unimodular $\boldsymbol{U}$.

Proof: Let $\boldsymbol{Y}=\boldsymbol{M P}=\boldsymbol{L} \boldsymbol{Q}$ be an lcrm $(\boldsymbol{M}, \boldsymbol{L})$, so $\boldsymbol{P}$ and $Q$ are right coprime (Theorem 1). Since $\boldsymbol{M L}=\boldsymbol{L M}$ is a $\mathrm{crm}(\boldsymbol{M}, \boldsymbol{L})$, by the definition of Icrm, $\boldsymbol{M L}=\boldsymbol{L M}=$ $\boldsymbol{M P X}=\boldsymbol{L} \boldsymbol{Q X}$ for some integer matrix $\boldsymbol{X}$. We then have $\boldsymbol{L}$ $=\boldsymbol{P X}$ and $\boldsymbol{M}=\boldsymbol{Q X}$. Because $\boldsymbol{P}$ and $\boldsymbol{Q}$ are right coprime, $\boldsymbol{X}$ is a gcrd $(\boldsymbol{M}, \boldsymbol{L})$. Clearly, $\boldsymbol{Y} \boldsymbol{X}=\boldsymbol{M L}$. We know any $\operatorname{lcrm}(M, L)$ is a right associate of $\boldsymbol{Y}$ and any $\operatorname{gcrd}(M, L)$ is a left associate of $\boldsymbol{X}$. So, we have $\operatorname{lcrm}(\boldsymbol{M}, \boldsymbol{L}) \cdot \boldsymbol{U}$. $\operatorname{gcrd}(\boldsymbol{M}, \boldsymbol{L})=\boldsymbol{M L}$ for some unimodular $\boldsymbol{U} . \triangle \triangle \triangle$

\section{MD Delay-Chain Systems}

A 1D delay-chain system as shown in Fig. 4 is a perfect reconstruction (PR) system, i.e., $\hat{x}(n)=x(n)$, iff $M$ and $L$ are coprime. This is shown in [24], and applications of this PR system in the design of filter bank systems can also be found therein. We shall now extend this concept to the MD case. One potential application of MD delaychain systems is to design MD filter banks where the analysis and synthesis filters have a certain symmetry. The research about such symmetry is still in progress.

A MD delay-chain system is shown in Fig. 11. (The case of $L=I$ is most commonly used and is a trivial PR filter bank.) We can see that this is a very special case of MD maximally decimated filter bank [8], [2] with $J(\boldsymbol{M})$ channels, where the analysis and synthesis filters are only shift operators (sometimes called "delays") defined as

$$
H_{k}(z)=z^{-L k} \quad \text { and } \quad F_{k}(z)=z^{L k} \quad \text { for } k \in \mathfrak{N}(M) \text {. }
$$

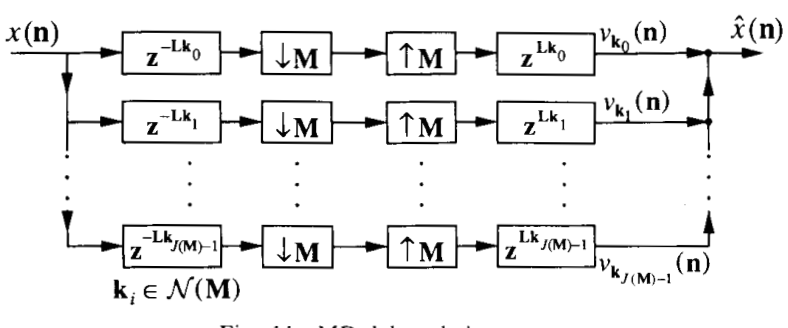

Fig. 11. MD delay-chain system.

As before, we assume $\boldsymbol{M}$ and $\boldsymbol{L}$ are nonsingular to avoid degeneracy. Clearly, Fig. 11 is an extension of the $1 D$ delay-chain system.

Using the definitions of decimation and expansion, we can write the signal $v_{\boldsymbol{k}}(\boldsymbol{n})$ in Fig. 11 for every $\boldsymbol{k} \in \mathfrak{N}(\boldsymbol{M})$ as follows:

$$
v_{k}(n)= \begin{cases}x(n) & n+L k \in \operatorname{LAT}(M) \\ 0 & \text { otherwise. }\end{cases}
$$

Define $\mathscr{L}_{\boldsymbol{k}} \triangleq\{\boldsymbol{n} \mid \boldsymbol{n}+\boldsymbol{L} \boldsymbol{k} \in \operatorname{LAT}(\boldsymbol{M})\}$. Clearly, $\mathscr{L}_{\boldsymbol{k}}$ is obtained by shifting $\operatorname{LAT}(\boldsymbol{M})$ by $-\boldsymbol{L} \boldsymbol{k}$. We can then redraw Fig. 11 as in Fig. 12. From Fig. 12, we can see that $\hat{x}(\boldsymbol{n})=x(\boldsymbol{n}) \forall \boldsymbol{n}$ iff

$$
\bigcup_{k \in \mathfrak{N}(\boldsymbol{M})} \mathfrak{L}_{k}=\mathscr{N}, \quad \text { and } \bigcap_{k \in \mathfrak{N}(\boldsymbol{M})} \mathfrak{L}_{k}=\text { empty set. }
$$

It can be verified that the above condition is true iff

$$
\mathcal{S} \triangleq\left\{((\boldsymbol{L} k))_{M} \mid \boldsymbol{k} \in \mathfrak{N}(\boldsymbol{M})\right\}=\mathscr{N}(\boldsymbol{M}) .
$$

From the definition of the modulo notation for integer vectors, all the elements in $S$ are also in $\mathscr{K}(\boldsymbol{M})$. Therefore, $(5.4)$ is true iff all $((\boldsymbol{L} k))_{M}$ 's (for $k \in \mathfrak{N}(\boldsymbol{M})$ ) are distinct. We now present the following sufficient condition for PR.

Theorem 5: If $\boldsymbol{L M} \stackrel{R}{=} \mathrm{lcrm}(\boldsymbol{L}, \boldsymbol{M})$, the MD delay chain is PR.

Proof: Suppose $\boldsymbol{L} \boldsymbol{M} \stackrel{R}{=} \operatorname{lcrm}(\boldsymbol{L}, \boldsymbol{M})$, but the MD delay chain is not PR, i.e., there exist two different $\boldsymbol{k}_{1}, \boldsymbol{k}_{2} \in$ $\mathfrak{N}(\boldsymbol{M})$ such that $\left(\left(\boldsymbol{L} \boldsymbol{k}_{1}\right)\right)_{\boldsymbol{M}}=\left(\left(\boldsymbol{L} \boldsymbol{k}_{2}\right)\right)_{\boldsymbol{M}}$. So, $\boldsymbol{L}\left(\boldsymbol{k}_{1}-\boldsymbol{k}_{2}\right)=$ $\boldsymbol{M n}$ for some $\boldsymbol{n} \in \mathfrak{N}$. Let $\boldsymbol{k}=\boldsymbol{k}_{1}-\boldsymbol{k}_{2}$. Since LAT $(\boldsymbol{L M})$ $=\operatorname{LAT}(\operatorname{lcrm}(\boldsymbol{L}, \boldsymbol{M}))=\operatorname{LAT}(\boldsymbol{L}) \cap \operatorname{LAT}(\boldsymbol{M})$ (from the Appendix), the above implies $\boldsymbol{L k}=\boldsymbol{M n}=\boldsymbol{L M} \boldsymbol{n}^{\prime}$ for some $\boldsymbol{n}^{\prime} \in \mathfrak{N}$. So, $\boldsymbol{k}=\boldsymbol{M} \boldsymbol{n}^{\prime}$. Because $\boldsymbol{k}_{1}, \boldsymbol{k}_{2} \in \mathfrak{N}(\boldsymbol{M})$, we can let $\boldsymbol{k}_{i}=\boldsymbol{M} \boldsymbol{y}_{i}, i=1,2$, where $\boldsymbol{y}_{i} \in[0,1)_{D}$. So, $\boldsymbol{k}=\boldsymbol{M y}$, where $\boldsymbol{y}=\boldsymbol{y}_{1}-\boldsymbol{y}_{2} \in(-1,1)^{D}$. Together with $\boldsymbol{k}=\boldsymbol{M n}^{\prime}$, we thus conclude that $\boldsymbol{y}=\boldsymbol{n}^{\prime}=\boldsymbol{k}=\mathbf{0}$ (0 stands for the zero vector), so $\boldsymbol{k}_{1}=\boldsymbol{k}_{2}$, which leads to contradiction.

However, $\boldsymbol{L M} \stackrel{R}{=} \operatorname{lcrm}(\boldsymbol{L}, \boldsymbol{M})$ is not a necessary condition. An example is

$$
\boldsymbol{M}=\left[\begin{array}{rr}
1 & -1 \\
1 & 2
\end{array}\right] \quad \text { and } \boldsymbol{L}=\left[\begin{array}{ll}
1 & 0 \\
0 & 2
\end{array}\right]
$$

It is easily checked that the above choice satisfies $\left\{((\boldsymbol{L} k))_{\boldsymbol{M}} \mid \boldsymbol{k} \in \mathfrak{N}(\boldsymbol{M})\right\}=\mathfrak{N}(\boldsymbol{M})$ (so the system is PR), 


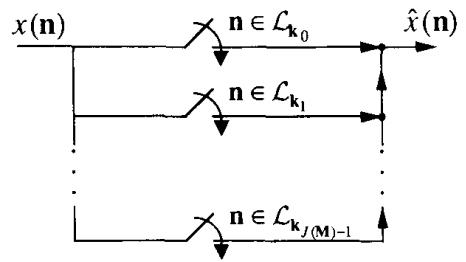

Fig. 12. MD delay-chain system redrawn

but

$$
\boldsymbol{L} \boldsymbol{M}=\left[\begin{array}{rr}
1 & -1 \\
2 & 4
\end{array}\right]
$$

is not even an rm of $\boldsymbol{M}$, since $\boldsymbol{M}^{-1} \boldsymbol{L} \boldsymbol{M}$ is not an integer matrix. Furthermore, we can get PR even with some singular $L$ 's. For example, when

$$
\boldsymbol{M}=\left[\begin{array}{cc}
1 & -1 \\
1 & 2
\end{array}\right] \quad \text { and } \boldsymbol{L}=\left[\begin{array}{ll}
0 & 0 \\
0 & 2
\end{array}\right]
$$

we still have

$$
S=\left\{\left[\begin{array}{l}
0 \\
0
\end{array}\right],\left[\begin{array}{l}
0 \\
1
\end{array}\right],\left[\begin{array}{l}
0 \\
2
\end{array}\right]\right\}=\mathscr{N}(\boldsymbol{M}) .
$$

However, if we assume $\boldsymbol{M L}=\boldsymbol{L M}$, we can show the following.

Theorem 6: If $\boldsymbol{M L}=\boldsymbol{L M}$, then the condition $\boldsymbol{L M} \stackrel{R}{=}$ lcrm $(\boldsymbol{L}, \boldsymbol{M})$ becomes necessary and sufficient for Fig. 11 to be a PR system.

Proof: Clearly, the sufficiency follows from Theorem 5 . If $\boldsymbol{L M}$ is not an lcrm $(\boldsymbol{L}, \boldsymbol{M})$ (hence $\boldsymbol{L}$ and $\boldsymbol{M}$ are not right coprime by Theorem 4$)$, we will show that the delay-chain system is not PR. Suppose $M P=L Q$ is an Icrm $(\boldsymbol{L}, \boldsymbol{M})$. Using Lemma 3 , we know there exists a non-unimodular gerd $(\boldsymbol{L}, \boldsymbol{M})$, say $\boldsymbol{X}$, such that $\boldsymbol{M P X}=$ $\boldsymbol{L Q X}=\boldsymbol{M L}=\boldsymbol{L M}$. Since $\boldsymbol{X}$ is not unimodular, there exist a nonzero $\boldsymbol{k} \in \mathfrak{T}(X)$. Let $\boldsymbol{k}^{\prime}=\boldsymbol{Q} \boldsymbol{k}$, which is also nonzero. Clearly, $\boldsymbol{k}^{\prime} \in \mathfrak{N}(\boldsymbol{Q X})=\mathfrak{K}(\boldsymbol{M})$. Now, we have a nonzero $\boldsymbol{k}^{\prime} \in \mathfrak{X}(\boldsymbol{M})$ such that $\left(\left(\boldsymbol{L} \boldsymbol{k}^{\prime}\right)\right)_{\boldsymbol{M}}=((\boldsymbol{L} \boldsymbol{Q} \boldsymbol{k}))_{\boldsymbol{M}}=((\boldsymbol{M P k}))_{M}$ $=\mathbf{0}$. We conclude that the elements in $\delta$ are not distinct because $((\boldsymbol{L O}))_{M}$ is also zero. Hence, the delay chain is not a PR system.

$\triangle \triangle \triangle$

The condition $\boldsymbol{M L}=\boldsymbol{L} \boldsymbol{M}$ is obviously not a necessary assumption in applications. The problem of finding the necessary and sufficient conditions for PR delay-chain systems without assuming $\boldsymbol{M L}=\boldsymbol{L M}$ is still open.

\section{Periodicity Matrices of Decimated Signals}

It is well known that in $1 \mathrm{D}$, a signal with period $P$ is also periodic with period $P S$ where $S$ is any nonzero integer. In MD, a similar fact is true. Let $x(n)$ have periodicity matrix $P$. If $\boldsymbol{Q}=\boldsymbol{P S}$, i.e., $\boldsymbol{Q}$ is an $\mathrm{rm}$ of $\boldsymbol{P}, \boldsymbol{Q}$ is also a periodicity matrix of $\boldsymbol{x}(\boldsymbol{n})[25, \mathrm{p}$. 12]. This is easily verified using the definition of periodicity matrices. Since the periodicity matrix of an MD signal is not unique, we are usually interested in those with the smallest absolute determinant. (We exclude the case of singular periodicity matrices.)

We shall consider the following question: when an MD signal $x(\boldsymbol{n})$ with periodicity matrix $\boldsymbol{L}$ is decimated by $\boldsymbol{M}$, is the output $y(\boldsymbol{n})=x(\boldsymbol{M n})$ periodic? If yes, what is the periodicity matrix? In other words, given $x(\boldsymbol{n}+\boldsymbol{L k})=$ $x(\boldsymbol{n}), \forall \boldsymbol{k} \in \mathfrak{K}$, we want to find $\boldsymbol{P}$ such that $y(\boldsymbol{n}+\boldsymbol{P k})=$ $y(\boldsymbol{n}), \forall \boldsymbol{k} \in \mathfrak{X}$. Since $y(\boldsymbol{n}+\boldsymbol{P k})=x(\boldsymbol{M n}+\boldsymbol{M P k})$, we can see that $y(\boldsymbol{n}+\boldsymbol{P k})=y(\boldsymbol{n})$ if $\boldsymbol{M P k}=\boldsymbol{L} \boldsymbol{q}$ for some $\boldsymbol{q}$ $\in \mathfrak{N}$. Therefore, $\boldsymbol{P}$ is a periodicity matrix of $y(\boldsymbol{n})$ if

$\forall k \in \mathscr{N}, \quad \exists q \in \mathfrak{N}, \quad$ such that $\boldsymbol{M P k}=\boldsymbol{L q}$.

Let $\boldsymbol{k}=\boldsymbol{e}_{0}, \cdots, \boldsymbol{e}_{D-1}$ successively, where $\boldsymbol{e}_{i}$ 's are columns of the identity matrix $I$, and collect all the corresponding $\boldsymbol{q}_{0}, \cdots, \boldsymbol{q}_{D-1}$ to form the matrix $\boldsymbol{Q}$. We see that $(6.1)$ is equivalent to

$$
\boldsymbol{M P}=\boldsymbol{L Q} \quad \text { for some integer matrix } \boldsymbol{Q} .
$$

This equation clearly states that $\boldsymbol{M P}=\boldsymbol{L} \boldsymbol{Q}$ is a $\mathrm{crm}(\boldsymbol{M}$, $\boldsymbol{L})$. Thus, $\boldsymbol{P}$ is a periodicity matrix of $y(\boldsymbol{n})$ if $\boldsymbol{P}=\boldsymbol{M}^{-1}$ crm $(\boldsymbol{M}, \boldsymbol{L})$. Clearly, $\boldsymbol{P}$ is not unique. Moreover, using the notation of $1 \mathrm{crm}$, we can conclude that the nonsingular $\boldsymbol{P}$ which satisfies (6.2) with the smallest absolute determinant is $\boldsymbol{P}=\boldsymbol{M}^{-1} \mathrm{lcrm}(\boldsymbol{M}, \boldsymbol{L})$. Note that $(6.2)$ is a sufficient condition for $\boldsymbol{P}$ to be a periodicity matrix of $y(n)$. If further knowledge about $x(\boldsymbol{n})$ is available, a periodicity matrix with even smaller absolute determinant can be found.

\section{A. Case of Wide-Sense-Stationary Input}

The result for the statistical case is similar. We shall first define wide-sense stationary (WSS) and cyclo-widesense stationary (CWSS) for the MD statistical process (random signal) as follows:

Definition: Let $R_{x x}(\boldsymbol{n}, \boldsymbol{m})=E\left[x(\boldsymbol{n}) x^{*}(\boldsymbol{n}-\boldsymbol{m})\right]$ denote the autocorrelation function of an MD process. The process is said to be WSS if

1) $E[x(\boldsymbol{n})]$ is a constant.

2) $R_{r x}(\boldsymbol{n}, \boldsymbol{m})$ is only a function of $\boldsymbol{m}$.

Definition: The process $x(\boldsymbol{n})$ is said to be CWSS with periodicity matrix $L$ (denoted as (CWSS) $L$ ) if

1) $E[x(\boldsymbol{n})]=E[x(\boldsymbol{n}+\boldsymbol{L k})], \forall \boldsymbol{k} \in \mathfrak{N}$, i.e., $E[x(\boldsymbol{n})]$ is periodic with periodicity matrix $\boldsymbol{L}$.

2) $R_{x x}(\boldsymbol{n}, \boldsymbol{m})=R_{x x}(\boldsymbol{n}+L \boldsymbol{k}, \boldsymbol{m}), \forall \boldsymbol{k}, \boldsymbol{m} \in \mathfrak{N}$, i.e., $R_{x x}(\boldsymbol{n}$, $\boldsymbol{m})$ is periodic with respect to $\boldsymbol{n}$ with periodicity matrix $\boldsymbol{L}$.

It is clear from above definitions that if $x(n)$ is (CWSS $)_{L}$ with unimodular $\boldsymbol{L}, x(\boldsymbol{n})$ is in fact wide-sense stationary (WSS). Now, given that $x(\boldsymbol{n})$ is $(\mathrm{CWSS})_{L}$, let $y(\boldsymbol{n})=$ $x(M n)$. What can we say about the cyclostationarity of $y(\boldsymbol{n})$ ? We know $E[y(\boldsymbol{n})]=E[x(\boldsymbol{M n})]$ and $R_{y y}(\boldsymbol{n}, \boldsymbol{m})=$ $E\left[y(\boldsymbol{n}) y^{*}(\boldsymbol{n}-\boldsymbol{m})\right]=E\left[x(\boldsymbol{M n}) x^{*}(\boldsymbol{M n}-\boldsymbol{M m})\right]=\boldsymbol{R}_{x x}(\boldsymbol{M n}$, $\boldsymbol{M m})$. That is, $\boldsymbol{E}[\boldsymbol{y}(\boldsymbol{n})]$ can be obtained by $\boldsymbol{M}$-fold decimating $E[x(n)]$, and $R_{w}(\boldsymbol{n}, \boldsymbol{m})$ can be obtained by $\boldsymbol{M}$-fold decimating $R_{x \mathrm{r}}(\boldsymbol{n}, \boldsymbol{m})$ with respect to both $\boldsymbol{n}$ and $\boldsymbol{m}$. Using the result we obtained for the deterministic case, we can conclude that $E[y(\boldsymbol{n})]$ has periodicity matrix $\boldsymbol{P}=\boldsymbol{M}^{-1}$ Icrm $(\boldsymbol{M}, \boldsymbol{L})$, and $R_{y,}(\boldsymbol{n}, \boldsymbol{m})$ also has the same periodicity matrix with respect to $n$. (Note that the decimation with 


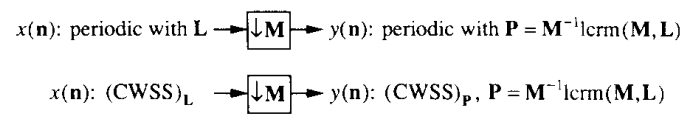

Fig. 13. Decimation of MD signals.

respect to the second argument $\boldsymbol{m}$ is not significant here.) Therefore, by the definition of CWSS, we know $y(n)$ is $(\mathrm{CWSS})_{\boldsymbol{P}}$, with $\boldsymbol{P}=\boldsymbol{M}^{-1} \operatorname{lcrm}(\boldsymbol{M}, \boldsymbol{L})$.

The above results are summarized in Fig. 13. Note that in $1 \mathrm{D}$, these simply reduce to $P=\operatorname{lcm}(M, L) / M=$ $L / \operatorname{gcd}(M, L)$ [32].

\section{B. Comments on Fundamental Periodicity Matrices}

As mentioned earlier, the periodicity matrix of an MD signal is not unique and we usually are interested in those with the smallest absolute determinant. For this reason, one defines the fundamental periodicity matrix as follows:

Definition: $\boldsymbol{P}_{0}$ is a fundamental periodicity matrix of $x(n)$ if

i) $\boldsymbol{P}_{0}$ is a periodicity matrix of $x(\boldsymbol{n})$.

ii) Any other periodicity matrix of $x(\boldsymbol{n})$, say $\boldsymbol{P}$, can be written as $\boldsymbol{P}=\boldsymbol{P}_{0} \boldsymbol{S}$ for some integer matrix $\boldsymbol{S}$. That is, $\boldsymbol{P}_{0}$ is a left divisor of all the periodicity matrices of $x(n)$.

It is clear from the above definition that a fundamental periodicity matrix of an MD signal is a periodicity matrix with the smallest absolute determinant (with singular periodicity matrices excluded), and is unique up to postmultiplication by a unimodular matrix. The existence of a fundamental periodicity matrix is assured by the following lemma:

Lemma 4: If $\boldsymbol{P}$ and $\boldsymbol{P}^{\prime}$ are both periodicity matrices of $x(\boldsymbol{n})$, then gcld $\left(\boldsymbol{P}, \boldsymbol{P}^{\prime}\right)$ is also a periodicity matrix.

Proof: Let $\boldsymbol{R}$ denote a gcld $\left(\boldsymbol{P}, \boldsymbol{P}^{\prime}\right)$. There exist integer matrices $\boldsymbol{A}$ and $\boldsymbol{B}$ such that $\boldsymbol{P A}+\boldsymbol{P}^{\prime} \boldsymbol{B}=\boldsymbol{R}$ (extension of the Euclid's theorem). We then have

$x(\boldsymbol{n}+\boldsymbol{R} \boldsymbol{k})=x(\boldsymbol{n}+\boldsymbol{P} \underbrace{\boldsymbol{A k}}_{\boldsymbol{k}^{\prime}}+\boldsymbol{P}^{\prime} \underbrace{\boldsymbol{B} \boldsymbol{k})}_{\boldsymbol{k}^{\prime \prime}}=x(\boldsymbol{n})$

So, $\boldsymbol{R}$ is also a periodicity matrix of $x(\boldsymbol{n})$.

From the above lemma, we can conclu given MD signal, a gcld of all the periodicity matrices is indeed a fundamental periodicity matrix. Since a gcld exists, the existence of a fundamental periodicity matrix is also guaranteed.

\section{Concluding Remarks}

In this paper, we have formulated and solved various theoretical issues in multidimensional (MD) multirate signal processing, including: the MD polyphase implementation technique for rational sampling rate alterations, the perfect reconstruction properties for the MD delay-chain systems, and the periodicity matrices of decimated MD signals (both deterministic and statistical). We have shown that all these can be solved with the help of the concepts of gcrd, geld, lcrm, and lclm, and other related properties for integer matrices. Although we are only interested in integer matrices, all the properties used here also apply to a more general kind of matrices, viz., matrices with elements in a principle ideal domain.

\section{Emerging Results from Other Authors}

After we had submitted this paper for review in August 1991 , we came to realize that the commutativity of MD decimators and expanders was also being considered by several other research groups. This commutativity problem has simultaneously been solved by different groups to different degrees.

Evans, McClellan, and Bamberger found the necessary and sufficient conditions of the commutativity to be: i) $\boldsymbol{M L}=\boldsymbol{L} \boldsymbol{M}$ and ii) $\operatorname{lcrm}(\boldsymbol{M}, \boldsymbol{L})=\boldsymbol{M L} \boldsymbol{V}$ where $\boldsymbol{V}$ is a unimodular matrix [33]. Also, through electronic mail correspondences, we realized that J. A. Sjogren (AFOSR) has also found similar conditions: i) $\boldsymbol{M L}=\boldsymbol{L} \boldsymbol{M}$ and ii) the absolute determinant of the generating matrix of the intersection lattice LAT $(\boldsymbol{M}) \cap$ LAT $(\boldsymbol{L})$ equals $J(\boldsymbol{M}) J(\boldsymbol{L})$.

A similar, but more relaxed, commutativity has also been considered. This is shown in Fig. 14. Gopinath and Burrus found that when $\boldsymbol{M}$ and $\boldsymbol{L}$ are left coprime, there exist $\boldsymbol{M}^{\prime}$ and $\boldsymbol{L}^{\prime}$, which are right coprime, such that the system in Fig. 14(a) is equivalent to the one in Fig. 14(b) [34]. Also, Kalker found that if $\boldsymbol{M}$ and $\boldsymbol{L}$ are such that the absolute determinant of the generating matrix of the intersection lattice LAT $(\boldsymbol{M}) \cap$ LAT $(\boldsymbol{L})$ equals $J(\boldsymbol{M}) J(\boldsymbol{L})$, then there exist $M^{\prime}$ and $L^{\prime}$ such that Figs. 14(a) and (b) are equivalent [35]. Inspired by this relaxed commutativity, we came up with the following lemma related to MFD's.

Lemma 5: Given matrices $\boldsymbol{M}$ and $\boldsymbol{L}$, we can always find $M^{\prime}$ and $L^{\prime}$ such that Fig. 14(a) and Fig. 14(b) are equivalent by computing an irreducible right MFD of $\boldsymbol{L}^{-1} \boldsymbol{M}$, i.e., $\boldsymbol{L}^{-1} \boldsymbol{M}=\boldsymbol{M}^{\prime} \boldsymbol{L}^{\prime-1}$ and $\boldsymbol{M}^{\prime}$ and $\boldsymbol{L}^{\prime}$ are right coprime. Conversely, given matrices $\boldsymbol{M}^{\prime}$ and $\boldsymbol{L}^{\prime}$ which are right coprime, we can always find $M$ and $L$ such that Figs. 14(a) and (b) are equivalent by computing a left MFD of $M^{\prime} L^{\prime-1}$, i.e., $\boldsymbol{M}^{\prime} \boldsymbol{L}^{\prime-1}=\boldsymbol{L}^{-1} \boldsymbol{M}$.

Proof: By modifying the proof of Theorem 3, we can obtain the conditions for Figs. 14(a) and (b) to be equivalent: i) $\boldsymbol{M} \boldsymbol{L}^{\prime}=\boldsymbol{L} \boldsymbol{M}^{\prime}$ and ii) $\boldsymbol{M} \boldsymbol{L}^{\prime}$ (or $\boldsymbol{L} \boldsymbol{M}^{\prime}$ ) is an lcrm $(\boldsymbol{M}, \boldsymbol{L})$. Combined with Theorem 1 , these conditions are equivalent to: i) $\boldsymbol{M} \boldsymbol{L}^{\prime}=\boldsymbol{L} \boldsymbol{M}^{\prime}$ and ii) $\boldsymbol{M}^{\prime}$ and $\boldsymbol{L}^{\prime}$ are right coprime. Then, the above lemma follows. $\triangle \triangle \triangle$

Remark: It turns out that we can use this relaxed commutativity in the RPI technique. Then, whenever $\boldsymbol{M}$ and $\boldsymbol{L}$ are left coprime, the RPI technique works.

It can be verified that all the above mentioned results are consistent with our results. Comparison of all these results leads to the conclusion that our conditions for commutativity ( $\boldsymbol{M L}=\boldsymbol{L} \boldsymbol{M}$ and $\boldsymbol{M}$ and $\boldsymbol{L}$ are coprime) are very explicit and easy to test. 


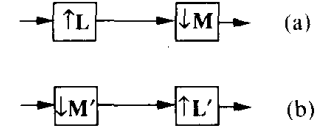

Fig. 14. Relaxed commutativity.

\section{APPENDIX}

In this Appendix, we will prove that $\mathrm{LAT}(\boldsymbol{L}) \cap \mathrm{LAT}$ $(\boldsymbol{M})=\mathrm{LAT}(\mathrm{Icrm}(\boldsymbol{M}, \boldsymbol{L}))$. A similar statement can be found in $[14$, p. 38, theorem 24.2] (although the $\mathrm{lcm}$ was mistaken to be $g c d$, which might be due to a typographical error in [14]). Since the proof of this statement was omitted in [14], we will now provide a formal proof.

The fact that the intersection of two lattices is also a lattice (which was called the greatest common submodul in [14] and the least common sublattice in [3]) is itself a nontrivial issue. To show this, we need the following theorem [27], [16].

Theorem A1: A set $\nabla$ of vectors in the $D$-dimensional space is a lattice iff it satisfies all the following three conditions:

1) If $a \in \mathcal{V}$ and $b \in \mathcal{V}$, then $a \pm b \in \mathcal{V}$.

2) $\checkmark$ contains $D$ linearly independent vectors.

3) There exists a positive number $\eta$ such that the zero vector is the only vector in $\nabla$ with norm less than $\eta$.

We use this theorem to show that $v=\operatorname{LAT}(\boldsymbol{L}) \cap$ LAT $(\boldsymbol{M})$ is indeed a lattice. Clearly, $\nabla$ satisfies Condition 1. To show that it satisfies Condition 2, we consider any nonsingular crm of $\boldsymbol{M}$ and $\boldsymbol{L}$ (for example, $J(\boldsymbol{M}) \boldsymbol{L}$ ), say $\boldsymbol{K}$. It is easily verified that all the column vectors in $K$ are in $\nabla$ and independent. It is also clear that $\nabla$ satisfies Condition 3 for any $0<\eta<1$, since all vectors in $\nabla$ have integer elements.

We will also need the following lemma (a similar statement can be found in $[14$, p. 38, theorem 24.1]):

Lemma $A$ l: $\operatorname{LAT}(X) \subset \operatorname{LAT}(\boldsymbol{Y})$, i.e., $\operatorname{LAT}(\boldsymbol{X})$ is a sublattice of LAT ( $\boldsymbol{Y})$ iff $\boldsymbol{X}=\boldsymbol{Y P}$ for some integer matrix $P$, i.e., $\boldsymbol{X}$ is an $\mathrm{rm}$ of $\boldsymbol{Y}$.

Proof:

1) If $\operatorname{LAT}(\boldsymbol{X}) \subset \operatorname{LAT}(\boldsymbol{Y})$, we have

$$
\forall k \in \mathfrak{N}, \quad \exists \boldsymbol{\exists} \in \mathfrak{N}, \quad \text { such that } X \boldsymbol{k}=Y \boldsymbol{p} .
$$

Let $\boldsymbol{k}=\boldsymbol{e}_{0}, \cdots, \boldsymbol{e}_{D-1}\left(\boldsymbol{e}_{i}\right.$ 's are same as in Section VI) and collect all the corresponding $p_{0}, \cdots, p_{D-1}$ to form the matrix $\boldsymbol{P}$, we get $\boldsymbol{X}=\boldsymbol{Y P}$.

2) Suppose $\boldsymbol{X}=\boldsymbol{Y P}$. Then, $\boldsymbol{x} \in \operatorname{LAT}(\boldsymbol{X}) \Rightarrow \boldsymbol{x}=\boldsymbol{X n}$ for some integer $\boldsymbol{n} \Rightarrow \boldsymbol{x}=\boldsymbol{Y P \boldsymbol { P }}$. Since $\boldsymbol{P} \boldsymbol{n}$ is also an integer vector, $\boldsymbol{x} \in \mathrm{LAT}(\boldsymbol{Y})$. Hence, we proved LAT $(\boldsymbol{X}) \subset \mathrm{LAT}$ $(\boldsymbol{Y})$. $\triangle \triangle \triangle$

We proceed to prove that LAT $(\boldsymbol{L}) \cap \mathrm{LAT}(\boldsymbol{M})=$ LAT $(\operatorname{lcrm}(\boldsymbol{M}, \boldsymbol{L}))$. Let LAT $(\boldsymbol{L}) \cap \operatorname{LAT}(\boldsymbol{M})=\mathrm{LAT}$ $(\boldsymbol{X})$. Because LAT $(\boldsymbol{X}) \subset \mathrm{LAT}(\boldsymbol{M}), \boldsymbol{X}=\boldsymbol{M} \boldsymbol{P}$ for some integer matrix $P$. Similarly, $X=L Q$ for some integer ma- trix $Q$. So, $X$ is a crm $(M, L)$ and hence an $\operatorname{rm}$ of $\operatorname{lcrm}(M$, $L$ ). Using Lemma A1, we can conclude that LAT $(X) \subset$ LAT $(\operatorname{lcrm}(\boldsymbol{M}, \boldsymbol{L}))$. On the other hand, let $\boldsymbol{M P}=\boldsymbol{L} \boldsymbol{Q}$ be a $\mathrm{crm}(\boldsymbol{M}, \boldsymbol{L})$. Using Lemma A1, we know LAT $(\boldsymbol{M P})$ $\subset \operatorname{LAT}(\boldsymbol{M})$ and LAT $(\boldsymbol{L} \boldsymbol{Q}) \subset \operatorname{LAT}(\boldsymbol{L})$. So, we have LAT $(\operatorname{crm}(\boldsymbol{M}, \boldsymbol{L})) \subset$ LAT $(\boldsymbol{M}) \cap \operatorname{LAT}(\boldsymbol{L})$. Hence, in particular, LAT $(1 \mathrm{crm}(\boldsymbol{M}, \boldsymbol{L})) \subset \mathrm{LAT}(\boldsymbol{M}) \cap$ LAT $(\boldsymbol{L})$ $=\operatorname{LAT}(\boldsymbol{X})$. Therefore, $\operatorname{LAT}(\operatorname{lcrm}(\boldsymbol{M}, \boldsymbol{L}))=\operatorname{LAT}(\boldsymbol{X})$ $=\operatorname{LAT}(\boldsymbol{L}) \cap \operatorname{LAT}(\boldsymbol{M})$.

\section{REFERENCES}

[1] J. Kovačević and M. Vetterli, "The commutativity of up/downsampling in two dimensions," IEEE Trans. Inform. Theory, vol. 37, no. 3, pp. 695-698, May 1991.

[2] E. Viscito and J. P. Allebach, "The analysis and design of multidimensional FIR perfect reconstruction filter banks for arbitrary sampling lattices," IEEE Trans. Circuits Syst., vol. 38, no. 1, pp. 2941 , Jan. 1991

[3] E. Dubois, "The sampling and reconstruction of time-varying imagery with application in video systems," Proc. IEEE, vol. 73, pp. 502-522, Apr. 1985

[4] R. Ansari and C.-L. Lau, "Two-dimensional IIR filters for exact reconstruction in tree structured subband decomposition," Electron. Lett., vol. 23, pp. 633-634, June 1987.

[5] R. Ansari and S. H. Lee, "Two-dimensional nonrectangular interpolation, decimation, and filter banks," presented at the ICASSP 88, Apr. 1988.

[6] E. Viscito and J. P. Allebach, "Design of perfect reconstruction multidimensional filter banks using cascaded Smith form matrices," in Proc. 1988 Int. Symp. Circuits Syst., June 1988, pp. 831-834.

[7] G. Karlsson and M. Vetterli, "Theory of two-dimensional multirate filter banks," IEEE Trans. Acoust., Speech, Signal Processing, vol. 38, no. 6, pp. 925-937, June 1990

[8] P. P. Vaidyanathan, "Fundamentals of multidimensional multirate digital signal processing," Sadhana, vol. 15, pp. 157-176, Nov. 1990.

[9] P. P. Vaidyanathan, "The role of Smith-form decomposition of integer matrices in multidimensional multirate systems, " in Proc. 1991 Int. Conf. ASSP, Toronto, Canada, May 1991, pp. 1777-1780.

[10] P. P. Vaidyanathan, "New results in multidimensional multirate systems, "' in Proc. 1991 Int. Symp. Circuits Syst., Singapore, June 1991, pp. $468-471$.

[11] J. Kovačević and M. Vetterli, "Nonseparable multidimensional perfect reconstruction filter banks and wavelet bases for $\mathbb{R}^{n}$," IEEE Trans. Inform. Theory, vol. 38, no. 2, pp. 533-555, Mar. 1992.

[12] T. Chen and P. P. Vaidyanathan, "Commutativity of $D$-dimensiona decimation and expansion matrices, and application to rational decimation systems," in Proc. IEEE Int. Conf. Acoust., Speech, Signal Processing, vol. 4, pp. 637-640, San Francisco, Mar. 1992.

113] T. Chen and P. P. Vaidyanathan, "Least common right/left multiples of integer matrices and applications to multidimensional multirate systems," in Proc. IEEE Int. Symp. Circuits Syst., San Diego, CA, May 1992, pp. 935-938.

[14] C. C. MacDuffee, The Theory of Matrices. New York: Chelsea, 1946.

[15] G. D. Forney, Jr., "Convolutional codes I: Algebraic structure,' IEEE Trans. Inform. Theory, vol. IT-16, no. 6, pp. 720-738, Nov. 1970.

[16] M. Newman, Integral Matrices. New York: Academic, 1972.

[17] M. Vidyasagar, Control System Synthesis: A Factorization Approach. Cambridge, MA: M.I.T. Press, 1985.

[18] T. Kailath, Linear Systems. Englewood Cliffs, NJ: Prentice-Hall, 1980.

[19] R. M. Mersereau and T. C. Speake, "The processing of periodically sampled multidimensional signals," IEEE Trans. Acoust., Speech. Signal Processing, vol. 31, no. 1, pp. 188-194, Feb. 1983.

[20] M. Vetterli, J. Kovačević, and D. J. Le Gall, "Perfect reconstruction 
filter banks for HDTV representation and coding," in Signal Processing: Image Communication 2. Elsevier Science Publisher, 1990, pp. 349-363.

[21] P. P. Vaidyanathan, "Multirate digital filters, filter banks, polyphase networks, and applications: A tutorial," Proc. IEEE, pp. 56-93, Jan 1990.

[22] R. E. Crochiere and L. R. Rabiner, Multirate Digital Signal Processing. Englewood Cliffs, NJ: Prentice-Hall, 1983.

[23] C. -C. Hsiao, "Polyphase filter matrix for rational sampling rate con versions," in Proc. IEEE Int. Conf. ASSP, Dallas, Apr. 1987, pp. 2173-2176.

[24] T. Q. Nguyen and P. P. Vaidyanathan, "Maximally decimated perfect reconstruction FIR filter banks with pairwise mirror-image analysis (and synthesis) frequency responses," IEEE Trans. Acoust. Speech, Signal Processing, vol. 36, no. 5, pp. 693-705, May 1988

[25] D. E. Dudgeon and R. M. Mersereau, Multidimensional Digital Signal Processing. Englewood Cliffs, NJ: Prentice-Hall, 1984.

[26] M. Vetterli, "Multidimensional subband coding: some theory and algorithms," Signal Processing, vol. 6, no. 2, pp. 97-112. Feb. 1984.

[27] J. W. S. Cassels, An Introduction to the Geometry of Numbers. Berlin: Springer-Verlag, 1959.

[28] R. H. Bamberger, "The directional filter bank: A multirate filter bank for the directional decomposition of images," doctoral dissertation, Georgia Institute Technol., Nov. 1990.

[29] T. Chen and P. P. Vaidyanathan, "Recent developments in multidimensional multirate systems," IEEE Trans. Video Technol. to be published June 1993.

[30] F. R. Gantmacher, The Theory of Matrices, vol. I. New York Chelsea, 1977

[31] A. Guessoum and R. M. Mersereau, "Fast algorithms for the multidimensional discrete Fourier transform," IEEE Trans. Acoust. Speech, Signal Processing, vol. 34, no. 4, pp. 937-943, Aug. 1986.

[32] V. P. Sathe and P. P. Vaidyanathan, "Effects of multirate systems on the statistical properties of random signals," IEEE Trans. Signal Processing, vol. 41, no. 1, pp. 131-146, Jan. 1993.

[33] B. L. Evans, J. H. McClellan and R. H. Bamberger, "A symbolic algebra for linear multidimensional multirate systems, " presented at the 1992 Conf. Inform. Sci. Syst., Princeton, NJ, Mar. 1992.

[34] R. A. Gopinath and C. S. Burrus, "On upsampling, downsampling, and rational sampling rate filter banks," IEEE Trans. Signal Processing, submitted for publication.

[35] A. A. C. M. Kalker, "Commutativity of up/down sampling," Elec'tron. Lett., vol. 28, no. 6, pp. 567-569, Mar. 1992.

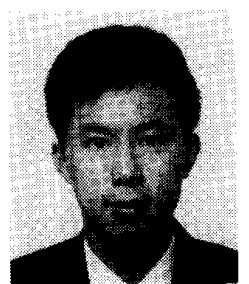

Tsuhan Chen ( $\mathrm{S}^{\prime} 90$ ) was born in Taipei, Taiwan, Republic of China, on November 7, 1965. He re ceived the B.S. degree in electrical engineering from the National Taiwan University, Taipei, Taiwan, in 1987, and the M.S. degree in electrica engineering from the California Institute of Technology in $1990 . \mathrm{He}$ is currently working towards the Ph.D. degree in electrical engineering at the California Institute of Technology. His main research interests are in digital signal processing. multirate filter banks, and multidimensional mul-

tirate systems.

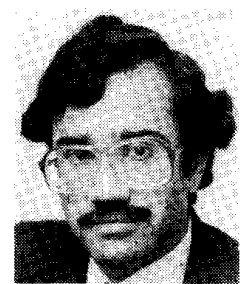

P. P. Vaidyanathan (S'80-M'83-SM'88-F'91) was born in Calcutta, India, on October 16, 1954 $\mathrm{He}$ received the B.Sc. (Hons.) degree in physics and the $B$.Tech. and $M$.Tech. degrees in radiophysics and electronics, all from the University of Calcutta, India, in 1974, 1977, and 1979, respectively, and the Ph.D degree in electrical and computer'engineering from the University of California, Santa Barbara, in 1982

He was a postdoctoral fellow at the University of California, Santa Barbara, from September 1982 to March 1983. In March 1983 he joined the Department of Electrica Engineering of the California Institute of Technology as an Assistant Professor, and since 1988 has been an Associate Professor of Electrical En gineering there. His main research interests are in digital signal processing. multirate systems, wavelet transforms, and adaptive filtering.

Dr. Vaidyanathan served as Vice Chairman of the Technical Program Committee for the 1983 IEEE International Symposium on Circuits and Systems, and as an Associate Editor for the IEEE TRAnsaCtIons on CiR. CUITS AND SySTEMS for the period 1985-1987. He also served as the Technical Program Chairman for the 1992 IEEE International Symposium on Circuits and Systems. He was a recipient of the Award for Excellence in Teaching at the California Institute of Technology for the year 1983-1984. He also received the NSF's Presidential Young Investigator Award in 1986. In 1989 he received the IEEE ASSP Senior Award for his paper on multirate perfect-reconstruction filter banks. In 1990 he received the S. K. Mitra Memorial Award from the Institute of Electronics and Telecommunication Engineers, India, for his joint paper in the IETE journal. He was elected a Fellow of the IEEE in 1991. 